\title{
GENERALIZED BLOCKS FOR SYMMETRIC GROUPS
}

\author{
BURKHARD KÜLSHAMMER, JøRN B. OLSSON, AND GEOFFREY R. ROBINSON
}

\begin{abstract}
We study, via character-theoretic methods, an $\ell$-analogue of the modular representation theory of the symmetric group, for an arbitrary integer $\ell \geq 2$. We find that many of the invariants of the usual block theory (ie. when $\ell$ is prime) generalize in a natural fashion to this new context.
\end{abstract}

The study of the modular representation theory of symmetric groups was initiated in the 1940's. One of the first highlights was the proof of the so-called Nakayama conjecture describing the distribution of the irreducible characters into $p$-blocks in terms of a combinatorial condition on the partitions labelling them. More specifically two irreducible characters are in the same $p$-block if and only if the partitions labelling them have the same $p$-core. There is also a comprehensive literature on decomposition numbers, Cartan matrices and other block-theoretic invariants of symmetric groups.

The representation theory of symmetric groups has served as a source of inspiration for the study of representations of other classes of groups and algebras. As an example we may refer to the book [9]. Corollary 5.38 in that book presents an analogue of the Nakayama conjecture for Iwahori-Hecke algebras for the symmetric group $S_{n}$ at an $\ell$-th root of unity. Donkin [4] has presented a direct link between the representation theory of these algebras and an $\ell$-analogue of the modular representation theory of the symmetric groups. It thus seems a natural problem to study " $\ell$-blocks" of $S_{n}$. We attempt to do this here based primarily on the ordinary character theory of symmetric groups and on some very general ideas from the character theory of finite groups. We study analogues of blocks, of the second main theorem on blocks, of decomposition matrices and of Cartan matrices in this context and prove an $\ell$-analogue of the Nakayama conjecture. We believe that this approach may provide additional insight, eg. concerning the invariant factors of Cartan matrices. For instance we show that these calculations for a given block of weight $w$ may be performed inside the wreath product $\mathbb{Z}_{\ell} \zeta S_{w}$. It should be mentioned that Brundan and Kleshchev [3] have recently given a formula for the determinant of the Cartan matrix of an $\ell$-block for the Hecke algebras. In view of [4] this also is the determinant of the Cartan matrix of an $\ell$-block of $S_{n}$. (See Proposition 6.10 for details).

The paper is organized as follows: The first two sections present a very general theory of contributions, perfect isometries, sections and blocks, suitable for our purposes. These sections may have independent interest beyond the questions at hand. In section 3 we introduce $\ell$-sections and $\ell$-blocks in symmetric groups and prove an analogue of the second main theorem of blocks. Then in section 4 we construct "basic sets", i.e. integral bases for the restrictions of the generalized

Subject classification [2000] Primary 20C30, 20C15, 20C20.

Corresponding author: Jørn B. Olsson, olsson@math.ku.dk. 
characters of $S_{n}$ to $\ell$-regular elements. Generalizing ideas of Osima we study in section 5 the equivalence of blocks of a given weight $w$. A relation between their decomposition matrices is given, showing also that their Cartan matrices have the same invariant factors. More generally, any such block is "perfectly isometric" to the set of irreducible characters of $\mathbb{Z}_{\ell} \prec S_{w}$ over a set of "regular" elements, defined by Osima. In the final section the invariant factors of the Cartan matrices are studied more closely. First the largest invariant factor is determined. In analogy with the prime case, each $\ell$-regular conjugacy class should contribute (in a quite subtle way) to the invariant factors and we make a specific conjecture what this contribution should be. Then we confirm (based on [3]) that the determinant of the $\ell$-Cartan matrix of $S_{n}$ is in accordance with a conjecture of Bessenrodt and Olsson [1], and we explain how our conjectured invariant factors predict the determinant.

\section{ON CONTRIBUTIONS AND ISOMETRIES}

Let $G$ be a finite group and $\operatorname{Irr}(G)$ denote its set of complex irreducible characters. Let $\mathcal{C}$ be a union of conjugacy classes of $G$. For complex-valued class functions $\alpha, \beta$ of $G$, we define

$$
\langle\alpha, \beta\rangle_{\mathcal{C}}:=\frac{1}{|G|} \sum_{y \in \mathcal{C}} \alpha(y) \overline{\beta(y)} .
$$

If $\langle\alpha, \beta\rangle_{\mathcal{C}}=0$, we say that $\alpha$ and $\beta$ are orthogonal across $\mathcal{C}$.

First we discuss linking of irreducible characters across $\mathcal{C}$. This notion has been considered by several authors in many contexts, but the original inspiration and motivation for considering it comes from the well-known fact that the usual $(p$-)blocks of irreducible characters of modular representation theory are precisely the $\mathcal{C}$-blocks (in the sense we will describe now) in the case that $\mathcal{C}$ is the set of $p$-regular elements of $G$.

If $\chi, \mu \in \operatorname{Irr}(G)$ are not orthogonal across $\mathcal{C}$, then they are said to be directly $\mathcal{C}$-linked. A subset $B$ of $\operatorname{Irr}(G)$ is said to be closed under $\mathcal{C}$-linking, if whenever $\chi \in B$ and $\mu \in \operatorname{Irr}(G)$ is directly $\mathcal{C}$-linked to $\chi$, then also $\mu \in B$. A $\mathcal{C}$-block of $G$ is a non-empty subset of $\operatorname{Irr}(G)$ which is minimal subject to being closed under $\mathcal{C}$-linking. Irreducible characters of $G$ in the same $\mathcal{C}$-block are said to be $\mathcal{C}$-linked.

For a complex-valued class function, $\theta$, of $G$, let $\theta^{\mathcal{C}}$ denote the class function of $G$ which agrees with $\theta$ on $\mathcal{C}$, and vanishes elsewhere. Similarly $\theta^{\mathcal{C}^{\prime}}$ is the class function which agrees with $\theta$ on $\mathcal{C}^{\prime}=G \backslash \mathcal{C}$ and vanishes on $\mathcal{C}$. It is an immediate consequence of the definition of $\mathcal{C}$-linking that whenever $\chi$ is an irreducible character of $G$, both $\chi^{\mathcal{C}}$ and $\chi^{\mathcal{C}^{\prime}}$ are linear combinations of irreducible characters from the same $\mathcal{C}$-block as $\chi$.

Our first general results will play a rôle in sections 2 and 4.

Lemma 1.1. Let $B$ be a $\mathcal{C}$-block of $G$. Then:

(1) Whenever $\sum_{\chi \in \operatorname{Irr}(G)} a_{\chi} \chi$ is a class function of $G$ which vanishes identically on $\mathcal{C}^{\prime}$, the class function $\sum_{\chi \in B} a_{\chi} \chi$ vanishes identically on $\mathcal{C}^{\prime}$.

(2) Whenever $\sum_{\chi \in \operatorname{Irr}(G)} a_{\chi} \chi$ is a class function of $G$ which vanishes identically on $\mathcal{C}$, the class function $\sum_{\chi \in B} a_{\chi} \chi$ vanishes identically on $\mathcal{C}$.

Proof: (1) Let $\mu$ be any irreducible character of $G$. If $\mu \notin B$ then $\left\langle\mu, \chi^{\mathcal{C}^{\prime}}\right\rangle=0$ for each $\chi \in B$, so that $\left\langle\sum_{\chi \in B} a_{\chi} \chi^{\mathcal{C}^{\prime}}, \mu\right\rangle=0$. On the other hand, if $\mu \in B$, then certainly $\left\langle\sum_{\chi \in \operatorname{Irr}(G)} a_{\chi} \chi^{\mathcal{C}^{\prime}}, \mu\right\rangle=0$, as, by hypothesis, $\sum_{\chi \in \operatorname{Irr}(G)} a_{\chi} \chi^{\mathcal{C}^{\prime}}=0$. Since 
$\left\langle\mu, \chi^{\mathcal{C}^{\prime}}\right\rangle=0$ for each $\chi \notin B$, we have $\left\langle\sum_{\chi \in B} a_{\chi} \chi^{\mathcal{C}^{\prime}}, \mu\right\rangle=0$. Hence $\sum_{\chi \in B} a_{\chi} \chi^{\mathcal{C}^{\prime}}=0$, as it is orthogonal to $\operatorname{Irr}(G)$. Thus $\sum_{\chi \in B} a_{\chi} \chi$ vanishes identically on $\mathcal{C}^{\prime}$. The proof of (2) is similar, and is omitted.

Corollary 1.2. Let $u \in \mathcal{C}$ and $v \in \mathcal{C}^{\prime}$. Then for each $\mathcal{C}$-block, $B$, of $G$, we have

$$
\sum_{\chi \in B} \chi\left(u^{-1}\right) \chi(v)=0 .
$$

This shows that $\mathcal{C}$-blocks separate $\mathcal{C}$ from $\mathcal{C}^{\prime}$. In fact, this could be used to give an alternative definition of $\mathcal{C}$-blocks: Any non-empty set of irreducible characters of $G$ which separates $\mathcal{C}$ from $\mathcal{C}^{\prime}$ is a union of $\mathcal{C}$-blocks (cf Osima's theorem in the usual block theory). We sketch a proof of this:

Assume that $S$ is a non-empty subset of $\operatorname{Irr}(\mathrm{G})$ such that

$$
\sum_{\chi \in S} \chi\left(u^{-1}\right) \chi(v)=0
$$

whenever $u \in \mathcal{C}$ and $v \in \mathcal{C}^{\prime}$. We claim that $S$ is a union of $\mathcal{C}$-blocks. Indeed, if we choose $y \in \mathcal{C}$, then the class function $\theta_{y}=\sum_{\chi \in S} \chi\left(y^{-1}\right) \chi$ vanishes identically on $\mathcal{C}^{\prime}$, by hypothesis. If $\mu \in \operatorname{Irr}(G) \backslash S$ then

$$
0=\left\langle\mu, \theta_{y}\right\rangle=\left\langle\mu^{\mathcal{C}}, \sum_{\chi \in S} \chi\left(y^{-1}\right) \chi\right\rangle=\sum_{\chi \in S} \chi(y)\left\langle\mu^{\mathcal{C}}, \chi\right\rangle .
$$

Since $y \in \mathcal{C}$ was arbitrary, it follows that $\sum_{\chi \in S}\left\langle\mu^{\mathcal{C}}, \chi\right\rangle \chi$ vanishes identically on $\mathcal{C}$. Write $\mu^{\mathcal{C}}=\alpha+\beta$ where $\alpha$ has all its irreducible constituents in $S$ and $\beta$ has all its irreducible constituents outside $S$. Then $\alpha=\sum_{\chi \in S}\langle\alpha, \chi\rangle \chi=\sum_{\chi \in S}\left\langle\mu^{\mathcal{C}}, \chi\right\rangle \chi$ vanishes identically on $\mathcal{C}$. Since $\mu^{\mathcal{C}}$ vanishes identically on $\mathcal{C}^{\prime}$, we see that $0=$ $\left\langle\alpha, \mu^{\mathcal{C}}\right\rangle=\langle\alpha, \alpha+\beta\rangle=\langle\alpha, \alpha\rangle$. Thus $\alpha=0$. Hence, for every $\chi \in S$, we have $0=\left\langle\mu^{\mathcal{C}}, \chi\right\rangle=\langle\mu, \chi\rangle_{\mathcal{C}}$. Thus no irreducible character in $S$ can be directly $\mathcal{C}$-linked to an irreducible character outside $S$, so that $S$ is a union of $\mathcal{C}$-blocks.

We next make some general observations about contributions.

Let $X(\mathcal{C})$ denote the part of the complex character table $X$ of $G$ consisting just of the columns corresponding to classes in $\mathcal{C}$. Hence $X(\mathcal{C})$ is a $k(G) \times k(\mathcal{C})$-matrix, where $k(G)$ is the number of conjugacy classes in $G$ and $k(\mathcal{C})$ is the number of conjugacy classes in $\mathcal{C}$.

Notice that $\Delta(\mathcal{C})=\overline{X(\mathcal{C})}^{t} X(\mathcal{C})$ is a diagonal matrix whose $i$-th diagonal entry is $\left|C_{G}\left(y_{i}\right)\right|$, where $y_{i}$ is a representative for the $i$-th conjugacy class in $\mathcal{C}$.

Let $\Gamma(\mathcal{C})$ denote the matrix of $\mathcal{C}$-contributions. This is the square $k(G) \times k(G)$ matrix with $(i, j)$-entry $\left\langle\chi_{i}, \chi_{j}\right\rangle_{\mathcal{C}}$, where $\chi_{i}, \chi_{j} \in \operatorname{Irr}(G)$.

We see immediately from the definition that

$$
\Gamma(\mathcal{C})=X(\mathcal{C}) \Delta(\mathcal{C})^{-1} \overline{X(\mathcal{C})}^{t} .
$$

Let $\left\{\phi_{r} \mid 1 \leq r \leq k(\mathcal{C})\right\}$ be any $\mathbb{C}$-basis for the space of complex-valued class functions of $G$ which vanish identically on $\mathcal{C}^{\prime}$. Let $\Phi(\mathcal{C})$ be the (invertible) $k(\mathcal{C}) \times$ $k(\mathcal{C})$-matrix with $(i, j)$-entry $\phi_{i}\left(y_{j}\right)$. Let $D(\mathcal{C})$ be the associated $k(G) \times k(\mathcal{C})$-matrix of coefficients (analogous to the "decomposition matrix" of the usual block theory) such that $X(\mathcal{C})=D(\mathcal{C}) \Phi(\mathcal{C})$.

Now we have

$$
\Gamma(\mathcal{C})=X(\mathcal{C}) \Delta(\mathcal{C})^{-1} \overline{X(\mathcal{C})}^{t},
$$


which re-arranges to $D(\mathcal{C}) C(\mathcal{C})^{-1} \overline{D(\mathcal{C})}^{t}$, where $C(\mathcal{C})=\overline{D(\mathcal{C})}^{t} D(\mathcal{C})$ is analogous to the Cartan matrix of the usual block theory (however, in our later work, we prefer to reserve the term "Cartan matrix" for the case when $D$ is integral and the $\phi_{i}$ 's are generalized characters).

This yields two expressions for the matrix of $\mathcal{C}$-contributions which will be useful later:

$$
\Gamma(\mathcal{C})=D(\mathcal{C}) C(\mathcal{C})^{-1} \overline{D(\mathcal{C})}^{t}=X(\mathcal{C})\left[\overline{X(\mathcal{C})}^{t} X(\mathcal{C})\right]^{-1} \overline{X(\mathcal{C})}^{t} .
$$

We note that $\Gamma(\mathcal{C})$ is idempotent and that $($ since $\Gamma(\mathcal{C}) X(\mathcal{C})=X(\mathcal{C}))$ its rank (and trace) is $k(\mathcal{C})$.

We say that the union of conjugacy classes $\mathcal{C}$ is closed if whenever $x \in \mathcal{C}$, every generator of $\langle x\rangle$ is also in $\mathcal{C}$. For the rest of this section we consider only closed unions of conjugacy classes. When $\mathcal{C}$ is a closed union of classes, $\mathcal{R}(\mathcal{C})$ denotes the $\mathbb{Z}$-submodule of the space of complex class-functions of $G$ generated by $\left\{\chi^{\mathcal{C}} \mid \chi \in \operatorname{Irr}(G)\right\}$ and $\mathcal{P}(\mathcal{C})$ denotes the $\mathbb{Z}$-submodule of $\mathcal{R}(\mathcal{C})$ consisting of generalized characters. Notice that $|G| \mathcal{R}(\mathcal{C}) \subseteq \mathcal{P}(\mathcal{C}) \subseteq \mathcal{R}(\mathcal{C})$, so that $\mathcal{R}(\mathcal{C})$ and $\mathcal{P}(\mathcal{C})$ both have the same $\mathbb{Z}$-rank. A $\mathcal{C}$-basic set, or just a basic set if there is no danger of ambiguity about $\mathcal{C}$, is any $\mathbb{Z}$-basis for $\mathcal{R}(\mathcal{C})$.

The notion of a closed union of classes goes back to Suzuki. Its definition ensures that $\mathcal{P}(\mathcal{C})$ has $\mathbb{Z}$-rank $k(\mathcal{C})$, and that, furthermore, any $\mathcal{C}$-basic set remains linearly independent over $\mathbb{C}$, so may be chosen as the $\mathbb{C}$-basis $\left\{\phi_{r} \mid 1 \leq r \leq k(\mathcal{C})\right\}$ used earlier (we will always make such a choice of basis when $\mathcal{C}$ is closed). For the convenience of the reader, let us briefly indicate a proof of this fact. We note that $\mathcal{C}^{\prime}$ is also a closed union of classes. Since $\left\{\chi^{\mathcal{C}} \mid \chi \in \operatorname{Irr}(G)\right\}$ spans a $\mathbb{C}$-subspace of dimension $k(\mathcal{C})$ of the space of complex-valued class functions of $G$, we certainly have $\operatorname{rk} \mathcal{R}(\mathcal{C}) \geq k(\mathcal{C})$. Similarly, $\operatorname{rk} \mathcal{R}\left(\mathcal{C}^{\prime}\right) \geq k\left(\mathcal{C}^{\prime}\right)$. On the other hand, it is easy to check that $|G| \mathcal{R}(G) \subseteq \mathcal{P}(\mathcal{C}) \oplus \mathcal{P}\left(\mathcal{C}^{\prime}\right) \subseteq \mathcal{R}(G)$, so that $\operatorname{rk} \mathcal{R}(\mathcal{C})+\operatorname{rk} \mathcal{R}\left(\mathcal{C}^{\prime}\right)=k(G)$.

Let $\operatorname{Cart}(\mathcal{C})$ denote the Abelian group $\mathcal{R}(\mathcal{C}) / \mathcal{P}(\mathcal{C})$ (which additionally has the structure of a commutative ring). We note that $\mathcal{R}(\mathcal{C})$ and $\mathcal{P}(\mathcal{C})$ are both free Abelian (as noted above, of rank $k(\mathcal{C})$ ). Also, $\operatorname{Cart}(\mathcal{C})$ is finite (of exponent dividing $|G|$ ) and can be generated by $k(\mathcal{C})$ or fewer elements. Now $\mathcal{R}(\mathcal{C}), \mathcal{P}(\mathcal{C})$ and $\operatorname{Cart}(\mathcal{C})$ all have a direct sum decomposition corresponding to the $\mathcal{C}$-blocks. Let $\operatorname{Cart}(\mathcal{C}, B)$ denote the summand corresponding to $B$, a union of $\mathcal{C}$-blocks (and similarly for other notations). Given a choice of $\mathbb{Z}$-basis for $\mathcal{P}(\mathcal{C}, B)$, say $\left\{\theta_{i} \mid 1 \leq i \leq s\right\}$, the Cartan matrix of $B$ is the $s \times s$ matrix $C(B)$ with $(i, j)$-entry $\left\langle\theta_{i}, \theta_{j}\right\rangle_{\mathcal{C}}$. A different choice of $\mathbb{Z}$-basis leads to a Cartan matrix $C^{\prime}(B)$ which satisfies $C^{\prime}(B)=A^{t} C(B) A$ for some unimodular integral matrix $A$, so the integral equivalence type of the associated quadratic form is well-defined (in particular, the invariant factors of the Cartan matrix are well-defined).

Let us now examine the relationship between the invariant factors of a Cartan matrix (in the sense above) and of the corresponding matrix of contributions.

Suppose that we have the contribution matrix $\Gamma(\mathcal{C})$ as before (we could also work with a union of $\mathcal{C}$-blocks, but we illustrate with the case of all of $\operatorname{Irr}(G))$. For ease of notation, let $s=k(\mathcal{C})$. By standard theory of finitely generated $\mathbb{Z}$-modules, there is a $\mathbb{Z}$-basis $\left\{\psi_{i} \mid 1 \leq i \leq s\right\}$, for $\mathcal{R}(\mathcal{C})$ such that $\left\{d_{i} \psi_{i} \mid 1 \leq i \leq s\right\}$ is a $\mathbb{Z}$-basis for $\mathcal{P}(\mathcal{C})$, and such that $d_{i}$ divides $d_{i+1}$ for all $i$ (the $d_{i}$ may be taken to be positive integers). Set $\beta_{i}=d_{i} \psi_{i}$ for each $i$. Notice, then, that $d_{s} \chi^{\mathcal{C}}$ has integral inner product with each element of $\mathcal{R}(\mathcal{C})$ for each $\chi \in \operatorname{Irr}(G)$. Furthermore, $d_{s}$ is the smallest positive 
integer with this property. For if $d$ is any positive integer with this property, then we see immediately that $d \theta$ is a generalized character for each $\theta \in \mathcal{R}(\mathcal{C})$. Hence the group $\operatorname{Cart}(\mathcal{C})$ has exponent dividing $d$. But the group $\operatorname{Cart}(\mathcal{C})$ visibly has exponent $d_{s}$, so $d_{s}$ divides $d$. We conclude that $d_{s}$ is the smallest positive integer for which $d_{s} \Gamma(\mathcal{C})(=M(\mathcal{C})$, say $)$, is an integral matrix.

We now claim that the non-zero invariant factors of $M(\mathcal{C})$ are precisely the $d_{s} / d_{i}$ $(1 \leq i \leq s)$ above. We recall that $\Gamma(\mathcal{C})$ has rank $k(\mathcal{C})$, which is also the rank of the Cartan matrix $C(\mathcal{C})$.

We notice that $\left\{d_{s} \chi^{\mathcal{C}} \mid \chi \in \operatorname{Irr}(G)\right\}$ generates $d_{s} \mathcal{R}(\mathcal{C})$, which clearly has $\mathbb{Z}$-basis $\left\{\frac{d_{s}}{d_{i}} \beta_{i} \mid 1 \leq i \leq s\right\}$.

The $i$-th row of $M(\mathcal{C})$ has $j$-th entry $d_{s}\left\langle\chi_{i}, \chi_{j}\right\rangle_{\mathcal{C}}$, where $\chi_{i}$ is the $i$-th irreducible character of $G$. It follows from the discussion above that we may perform invertible (integral) elementary row operations on $M(\mathcal{C})$ to obtain a new matrix $N(\mathcal{C})$ whose $i$-th row has $j$-th entry $\left\langle\frac{d_{s}}{d_{i}} \beta_{i}, \chi_{j}\right\rangle_{\mathcal{C}}$ (for $1 \leq i \leq k(\mathcal{C})$ ), and whose remaining rows are zero.

Now it is easily verified that there is a $\mathbb{Z}$-basis of $\mathcal{R}(\mathcal{C})$ which is "dual" to $\left\{\beta_{i} \mid 1 \leq\right.$ $i \leq s\}$, say $\left\{\gamma_{i} \mid 1 \leq i \leq s\right\}$, in the sense that $\left\langle\beta_{i}, \gamma_{j}\right\rangle=\delta_{i, j}$ for all $i, j$ (this basis is uniquely determined by $\left\{\beta_{i} \mid 1 \leq i \leq s\right\}$ ). By performing invertible (integral) elementary column operations on our matrix $N(\mathcal{C})$, we obtain a matrix with $(i, j)$ entry

$$
\left\langle\frac{d_{s}}{d_{i}} \beta_{i}, \gamma_{j}\right\rangle=\delta_{i, j} \frac{d_{s}}{d_{i}}
$$

for $1 \leq j \leq s$, and all other entries 0 . Conjugating by a suitable permutation matrix, we deduce

Lemma 1.3. In the above notation, the non-zero invariant factors of $M(\mathcal{C})$, listed in correct order, are $1, \frac{d_{s}}{d_{s-1}}, \ldots, \frac{d_{s}}{d_{2}}, \frac{d_{s}}{d_{1}}$.

We finally discuss the notion of perfect isometry in a somewhat more general context than the usual block-theoretic one. We remark however that, when specialized to the usual block-theoretic case, the notion we use here appears to be slightly weaker than M. Broué's definition (see eg. [2]). However, for the purposes of the invariants we will be interested in later, this definition is strong enough.

Suppose that $\mathcal{C}$ and $\mathcal{D}$ are closed unions of conjugacy classes of finite groups $G$ and $H$ respectively. Let $B=B(\mathcal{C})$ and $B^{\prime}=B^{\prime}(\mathcal{D})$ be sets of irreducible characters of $G, H$ which are closed under $\mathcal{C}$-linking and $\mathcal{D}$-linking respectively. Let $\Gamma(\mathcal{C}, B)$ and $\Gamma\left(\mathcal{D}, B^{\prime}\right)$ be the contribution matrices associated to $B$ and $B^{\prime}$ respectively. We say that $B$ and $B^{\prime}$ are perfectly isometric if there is a diagonal matrix of signs $S$ such that $S \Gamma(\mathcal{C}, B) S=\Gamma\left(\mathcal{D}, B^{\prime}\right)$.

Suppose then that $B$ and $B^{\prime}$ are perfectly isometric. Let $B=\left\{\chi_{i} \mid 1 \leq i \leq n\right\}$ and $B^{\prime}=\left\{\mu_{i} \mid 1 \leq i \leq n\right\}$. The relationship between the contribution matrices just means that there are signs $\varepsilon_{1}, \ldots, \varepsilon_{n}$ such that $\left\langle\chi_{i}, \chi_{j}\right\rangle_{\mathcal{C}}=\left\langle\varepsilon_{i} \mu_{i}, \varepsilon_{j} \mu_{j}\right\rangle_{\mathcal{D}}$ for all $i, j$.

We then have:

Proposition 1.4. (1) Under the above hypotheses, the Abelian groups $\mathcal{R}(\mathcal{C}, B)$ and $\mathcal{R}\left(\mathcal{D}, B^{\prime}\right)$ are isomorphic via an isomorphism which restricts to an isomorphism between $\mathcal{P}(\mathcal{C}, B)$ and $\mathcal{P}\left(\mathcal{D}, B^{\prime}\right)$. In particular, the Abelian groups Cart $(\mathcal{C}, B)$ and $\operatorname{Cart}\left(\mathcal{D}, B^{\prime}\right)$ are isomorphic.

(2) (With suitable choice of $\mathbb{Z}$-bases), the Cartan matrices $C(B)$ and $C\left(B^{\prime}\right)$ are equal. 
Proof: For $a_{1}, \ldots, a_{n} \in \mathbb{Z}$, the class function $\sum_{i=1}^{n} a_{i} \varepsilon_{i} \mu_{i}^{\mathcal{D}}$ is orthogonal to every $\mu \in \operatorname{Irr}(H) \backslash B^{\prime}$ while

$$
\left\langle\sum_{i=1}^{n} a_{i} \varepsilon_{i} \mu_{i}^{\mathcal{D}}, \mu_{j}\right\rangle=\left\langle\sum_{i=1}^{n} a_{i} \varepsilon_{i} \mu_{i}^{\mathcal{D}}, \mu_{j}^{\mathcal{D}}\right\rangle=\varepsilon_{j}\left\langle\sum_{i=1}^{n} a_{i} \chi_{i}^{\mathcal{C}}, \chi_{j}^{\mathcal{C}}\right\rangle=\varepsilon_{j}\left\langle\sum_{i=1}^{n} a_{i} \chi_{i}^{\mathcal{C}}, \chi_{j}\right\rangle
$$

for $j=1, \ldots, n$. Thus, if $\sum_{i=1}^{n} a_{i} \chi_{i}^{\mathcal{C}}$ is zero then $\sum_{i=1}^{n} a_{i} \varepsilon_{i} \mu_{i}^{\mathcal{D}}$ is orthogonal to every $\mu \in \operatorname{Irr}(H)$, so $\sum_{i=1}^{n} a_{i} \varepsilon_{i} \mu_{i}^{\mathcal{D}}$ is zero.

It follows that the map $\phi_{S}: \mathcal{R}(\mathcal{C}, B) \rightarrow \mathcal{R}\left(\mathcal{D}, B^{\prime}\right)$ sending $\sum_{i=1}^{n} a_{i} \chi_{i}^{\mathcal{C}}$ (where $\left.a_{1}, \ldots, a_{n} \in \mathbb{Z}\right)$ to $\sum_{i=1}^{n} a_{i} \varepsilon_{i} \mu_{i}^{\mathcal{D}}$ is well-defined. If $\sum_{i=1}^{n} a_{i} \chi_{i}^{\mathcal{C}} \in \mathcal{P}(\mathcal{C}, B)$ then, by the formula above, $\sum_{i=1}^{n} a_{i} \varepsilon_{i} \mu_{i}^{\mathcal{D}}$ has integral inner product with every $\mu \in \operatorname{Irr}(H)$, so $\sum_{i=1}^{n} a_{i} \varepsilon_{i} \mu_{i}^{\mathcal{D}} \in \mathcal{P}\left(\mathcal{D}, B^{\prime}\right)$.

Now it is clear that $\phi_{S}$ is invertible by similar arguments, and that its inverse maps $\mathcal{P}\left(\mathcal{D}, B^{\prime}\right)$ into $\mathcal{P}(\mathcal{C}, B)$. Hence $\phi_{S}$ yields the isomorphisms claimed in the statement of part (1) of the proposition. Since the isomorphism $\phi_{S}$ of part (1) restricts to an isomorphism of $\mathbb{Z}$-modules between $\mathcal{P}(\mathcal{C}, B)$ and $\mathcal{P}\left(\mathcal{D}, B^{\prime}\right)$ which also preserves inner products, part (2) follows immediately.

\section{ON SECTIONS AND BLOCKS}

In this section we formulate a generalized theory of sections and blocks for $G$. As well as defining blocks by linking, as discussed in Section 1, we wish to discuss blocks of centralizers of elements of certain distinguished conjugacy classes, and relate these to blocks of $G$, in (rather loose) analogy with the usual block theory.

Let $\mathcal{X}$ be a union of conjugacy classes, containing the identity, of our finite group $G$. Suppose that for each element $x \in \mathcal{X}$, there is a union of conjugacy classes $\mathcal{Y}(x)$, containing the identity, of the centralizer $C_{G}(x)$, such that two elements of $x \mathcal{Y}(x)$ are $G$-conjugate if and only if they are $C_{G}(x)$-conjugate, and such that $C_{G}(x y) \leq C_{G}(x)$ for each $y \in \mathcal{Y}(x)$. Suppose further that $\mathcal{Y}\left(x^{g}\right)=\mathcal{Y}(x)^{g}$ for all $x \in \mathcal{X}, g \in G$ and that $G$ is the disjoint union of the conjugacy classes $(x y)^{G}$, as $x$ runs through a set of representatives for the conjugacy classes in $\mathcal{X}$ and $y$ runs through a set of representatives for the $C_{G}(x)$-conjugacy classes of $\mathcal{Y}(x)$. For any $x \in \mathcal{X}$, we call the union of the $G$-conjugacy classes meeting $x \mathcal{Y}(x)$ the $\mathcal{Y}$-section of $x$. We will refer to $\mathcal{Y}\left(1_{G}\right)$ as the set of $\mathcal{Y}$-regular elements of $G$. We may consider $\mathcal{Y}(x)$ as the set of $\mathcal{Y}$-regular elements of $C_{G}(x)$ for each $x \in \mathcal{X}$.

Notice that, for each $x \in \mathcal{X}$, induction of complex class functions gives an isometry from the inner product space of class functions of $C_{G}(x)$ vanishing outside $x \mathcal{Y}(x)$ onto the space of class functions of $G$ vanishing outside the $\mathcal{Y}$-section of $x$.

We define an $(\mathcal{X}, \mathcal{Y})$-block of $G$ simply as a $\mathcal{Y}\left(1_{G}\right)$-block of $G$ in the sense of Section 1. Such a block separates $\mathcal{Y}\left(1_{G}\right)$ from its complement in $G$, by Corollary 1.2. However, we also wish to define $(\mathcal{X}, \mathcal{Y})$-blocks of $C_{G}(x)$ for each $x \in \mathcal{X}$. A non-empty set of irreducible characters of $C_{G}(x)$ which is minimal subject to separating $\mathcal{Y}(x)$ from its complement in $C_{G}(x)$ (which, as $x$ is central in $C_{G}(x)$, is equivalent to separating $x \mathcal{Y}(x)$ from its complement) is considered to be an $(\mathcal{X}, \mathcal{Y})$ block of $C_{G}(x)$. We note that, by the results of section 1, irreducible characters in different $(\mathcal{X}, \mathcal{Y})$-blocks of $C_{G}(x)$ are orthogonal across $x \mathcal{Y}(x)$, and this condition could equally well be used to define $(\mathcal{X}, \mathcal{Y})$-blocks of $C_{G}(x)$. 
Definitions. For an irreducible character $\chi$, of $G$ and $\beta$ a union of $(\mathcal{X}, \mathcal{Y})$-blocks of $C_{G}(x)$, we define the character $\chi^{(\beta)}$ of $C_{G}(x)$ via

$$
\chi^{(\beta)}=\sum_{\mu \in \beta}\left\langle\operatorname{Res}_{C_{G}(x)}^{G}(\chi), \mu\right\rangle \mu .
$$

Let $b$ be an $(\mathcal{X}, \mathcal{Y})$-block of $C_{G}(x)$. We say that an $(\mathcal{X}, \mathcal{Y})$-block $B$ of $G$ dominates $b$ (and write $B \geq b$ ) if there is some irreducible character $\chi \in B$ and some $y \in \mathcal{Y}(x)$ such that

$$
\left\langle\chi, \operatorname{Ind}_{C_{G}(x)}^{G}\left(\sum_{\mu \in b} \mu\left(x^{-1} y^{-1}\right) \mu\right)\right\rangle \neq 0 .
$$

Notice that we have

$$
\left\langle\chi, \operatorname{Ind}_{C_{G}(x)}^{G}\left(\sum_{\mu \in b} \mu\left(x^{-1} y^{-1}\right) \mu\right)\right\rangle=\chi^{(b)}(x y)
$$

for each $y \in \mathcal{Y}(x)$. Hence $B$ dominates $b$ if and only if there is some $\chi \in B$ such that $\chi^{(b)}$ does not vanish identically on $x \mathcal{Y}(x)$. Furthermore, we see that for each $y \in \mathcal{Y}(x)$, we have $\chi(x y)=\sum_{b} \chi^{(b)}(x y)$, where $b$ runs over $(\mathcal{X}, \mathcal{Y})$-blocks of $C_{G}(x)$ dominated by $B$. We note also that if $b^{\prime}$ is another $(\mathcal{X}, \mathcal{Y})$-block of $C_{G}(x)$, and $\gamma$ is an irreducible character of $G$ (possibly equal to $\chi$ ), then $\chi^{(b)}$ and $\gamma^{\left(b^{\prime}\right)}$ are orthogonal across $x \mathcal{Y}(x)$.

We say that the $(\mathcal{X}, \mathcal{Y})$-blocks of $G$ satisfy the Second Main Theorem property if for each $x \in \mathcal{X}$ and each $(\mathcal{X}, \mathcal{Y})$-block $b$ of $C_{G}(x), b$ is dominated by a unique $(\mathcal{X}, \mathcal{Y})$ block of $G$ (it is always the case that each $(\mathcal{X}, \mathcal{Y})$-block of $C_{G}(x)$ is dominated by at least one $(\mathcal{X}, \mathcal{Y})$-block of $G$ ).

Theorem 2.1. The $(\mathcal{X}, \mathcal{Y})$-blocks of $G$ satisfy the Second Main Theorem property, if and only if, whenever $x$ is an element of $\mathcal{X}$ and $B$ is an $(\mathcal{X}, \mathcal{Y})$-block of $G$, there is a union of $(\mathcal{X}, \mathcal{Y})$-blocks of $C_{G}(x)$, say $\beta(x, B)=\beta$, such that whenever $y, z$ are elements of $\mathcal{Y}(x)$, we have

$$
\sum_{\chi \in B} \chi\left(x^{-1} y^{-1}\right) \chi(x z)=\sum_{\mu \in \beta} \mu\left(y^{-1}\right) \mu(z)
$$

Proof: Suppose that $(\mathcal{X}, \mathcal{Y})$-blocks of $G$ have the property of the statement of the Theorem. Choose an element $x \in \mathcal{X}$ and an element $y \in \mathcal{Y}(x)$. Consider the class function

Notice that

$$
\theta_{x y}=I n d_{C_{G}(x)}^{G}\left(\sum_{\mu \in \beta} \mu\left(x^{-1} y^{-1}\right) \mu\right) .
$$

$$
\psi_{x y}=\sum_{\mu \in \beta} \mu\left(x^{-1} y^{-1}\right) \mu
$$

vanishes outside $x \mathcal{Y}(x)$, as $\beta$ is a union of $(\mathcal{X}, \mathcal{Y})$-blocks of $C_{G}(x)$, so that $\theta_{x y}$ vanishes outside the $\mathcal{Y}$-section of $x$ and has the same norm as $\psi_{x y}$. Furthermore, $\psi_{x y}$ and $\theta_{x y}$ agree on $x \mathcal{Y}(x)$.

Let $\Phi_{x y}=\sum_{\chi \in B} \chi\left(x^{-1} y^{-1}\right) \chi$. Notice that our hypotheses imply that

$$
\left\langle\Phi_{x y}, \Phi_{x y}\right\rangle=\sum_{\chi \in B}|\chi(x y)|^{2}=\sum_{\mu \in \beta}|\mu(x y)|^{2}=\left\langle\psi_{x y}, \psi_{x y}\right\rangle=\left\langle\theta_{x y}, \theta_{x y}\right\rangle .
$$

Furthermore, our hypotheses also imply that $\Phi_{x y}$ agrees with $\theta_{x y}$ on the $\mathcal{Y}$ section of $x$ in $G$. Since $\theta_{x y}$ vanishes identically outside the $\mathcal{Y}$-section of $x$ in $G$ and 
has the same norm as $\Phi_{x y}$, we must conclude that $\Phi_{x y}$ also vanishes outside the $\mathcal{Y}$-section of $x$, and that $\Phi_{x y}=\theta_{x y}$. Hence every irreducible character of $G$ outside $B$ is orthogonal to $\operatorname{Ind}_{C_{G}(x)}^{G}\left(\psi_{x y}\right)$.

Let $b_{1}, \ldots, b_{n}$ be the distinct $(\mathcal{X}, \mathcal{Y})$-blocks whose union is $\beta$. We now know that for each irreducible character $\gamma$ of $G$ outside $B$, and each $y \in \mathcal{Y}(x)$, we have

$$
\sum_{i=1}^{n} \gamma^{\left(b_{i}\right)}(x y)=\left\langle\gamma, \theta_{x y}\right\rangle=0 .
$$

Thus $\sum_{i=1}^{n} \gamma^{\left(b_{i}\right)}$ vanishes identically on $x \mathcal{Y}(x)$. Since the distinct $\gamma^{\left(b_{i}\right)}$ are mutually orthogonal across $x \mathcal{Y}(x)$, each of them vanishes identically on $x \mathcal{Y}(x)$. By definition of domination, this means that each $b_{i}$ is dominated by the $(\mathcal{X}, \mathcal{Y})$-block $B$ and no other.

On the other hand, for each irreducible character $\chi \in B$, we have

$$
\chi(x y)=\sum_{i=1}^{n} \chi^{\left(b_{i}\right)}(x y)\left(=\left\langle\chi, \Phi_{x y}\right\rangle\right)
$$

(for each $y \in \mathcal{Y}(x)$ ) so that $\chi^{(b)}$ vanishes identically on $x \mathcal{Y}(x)$ for each $(\mathcal{X}, \mathcal{Y})$-block of $C_{G}(x)$ which is not a subset of $\beta$. Hence each $(\mathcal{X}, \mathcal{Y})$-block of $C_{G}(x)$ dominated by $B$ is a subset of $\beta$.

Since each $(\mathcal{X}, \mathcal{Y})$-block of $C_{G}(x)$ is dominated by at least one $(\mathcal{X}, \mathcal{Y})$-block of $G$, we see that each $(\mathcal{X}, \mathcal{Y})$-block of $C_{G}(x)$ is a subset of $\beta(x, B)$ for precisely one $(\mathcal{X}, \mathcal{Y})$-block $B$ of $G$, and the $(\mathcal{X}, \mathcal{Y})$-blocks of $G$ satisfy the Second Main Theorem property.

The proof of the implication in the opposite direction is rather similar, so we only indicate it. For $x$ an element of $\mathcal{X}$ and $B$ an $(\mathcal{X}, \mathcal{Y})$-block of $G$, let $\beta=\beta(x, B)$ denote the union of those $(\mathcal{X}, \mathcal{Y})$-blocks of $C_{G}(x)$ dominated by $B$. Then we find that for each $y \in \mathcal{Y}(x)$, we have

$$
\sum_{\chi \in B} \chi\left(x^{-1} y^{-1}\right) \chi=\operatorname{Ind}_{C_{G}(x)}^{G}\left(\sum_{\mu \in \beta} \mu\left(x^{-1} y^{-1}\right) \mu\right),
$$

and evaluating both class functions at $x z$ for $z \in \mathcal{Y}(x)$ gives the result.

Corollary 2.2. The $(\mathcal{X}, \mathcal{Y})$-blocks of $G$ satisfy the Second Main Theorem property if and only if, for each $(\mathcal{X}, \mathcal{Y})$-block $B$ of $G$, there is for each $x \in \mathcal{X}$ a (possibly empty) union of $(\mathcal{X}, \mathcal{Y})$-blocks $\beta=\beta(x, B)$ of $C_{G}(x)$ such that for each irreducible character $\chi \in B$, and each irreducible character $\mu \in \beta(x, B)$ we may find a complex number $d_{\chi, \mu}$ such that for each $y \in \mathcal{Y}(x)$ we have

$$
\chi(x y)=\sum_{\mu \in \beta} d_{\chi, \mu} \mu(x y),
$$

and, furthermore, $\beta(x, B)$ and $\beta\left(x, B^{\prime}\right)$ are disjoint whenever $B$ and $B^{\prime}$ are distinct $(\mathcal{X}, \mathcal{Y})$-blocks of $G$.

Proof: Suppose that $(\mathcal{X}, \mathcal{Y})$-blocks of $G$ satisfy the Second Main Theorem property. Then whenever $B$ is an $(\mathcal{X}, \mathcal{Y})$-block of $G$, and $x \in \mathcal{X}$, we let $\beta=\beta(x, B)$ be the union of those $(\mathcal{X}, \mathcal{Y})$-blocks of $C_{G}(x)$ dominated by $B$. For each $\chi \in B$, we have (for each $y \in \mathcal{Y}(x)$ )

$$
\chi(x y)=\chi^{(\beta)}(x y),
$$


so complex numbers as in the statement of the corollary exist.

Conversely, suppose that for each $x \in \mathcal{X}$, and each $(\mathcal{X}, \mathcal{Y})$-block $B$ of $G$, we have a union of blocks $\beta=\beta(x, B)$ as in the statement. We claim that for each $\chi \in B$, we have $\chi^{(b)}=0$ unless the $(\mathcal{X}, \mathcal{Y})$-block $b$ of $C_{G}(x)$ is a subset of $\beta$. For, given such a block $b$ which is not a subset of $\beta$, the orthogonality of characters from different $(\mathcal{X}, \mathcal{Y})$-blocks of $C_{G}(x)$ across $x \mathcal{Y}(x)$, and the fact that we have

$$
\chi(x y)=\sum_{\mu \in \beta} d_{\chi, \mu} \mu(x y)
$$

for each $y \in \mathcal{Y}(x)$ shows that $\chi^{(b)}$ and $\operatorname{Res}_{C_{G}(x)}^{G}(\chi)$ are orthogonal across $x \mathcal{Y}(x)$. But $\chi^{(b)}$ is certainly orthogonal to $\operatorname{Res}_{C_{G}(x)}^{G}(\chi)-\chi^{(b)}$ across $x \mathcal{Y}(x)$, so $\chi^{(b)}$ is orthogonal to $\chi^{(b)}$ across $x \mathcal{Y}(x)$. In other words, $\chi^{(b)}$ vanishes identically on $x \mathcal{Y}(x)$.

We conclude that each $(\mathcal{X}, \mathcal{Y})$-block of $C_{G}(x)$ dominated by $B$ is a subset of $\beta$. Since the hypotheses we are assuming imply that $\beta(x, B)$ and $\beta\left(x, B^{\prime}\right)$ are disjoint whenever $B$ and $B^{\prime}$ are distinct, we conclude that each $(\mathcal{X}, \mathcal{Y})$-block of $C_{G}(x)$ is dominated by a unique $(\mathcal{X}, \mathcal{Y})$-block of $G$, so $(\mathcal{X}, \mathcal{Y})$-blocks of $G$ satisfy the Second Main Theorem property.

Corollary 2.3. Suppose that $(\mathcal{X}, \mathcal{Y})$-blocks of $G$ satisfy the Second Main Theorem property. Then:

(1) Irreducible characters of $G$ which are in different $(\mathcal{X}, \mathcal{Y})$-blocks are orthogonal across each $\mathcal{Y}$-section of $G$.

(2) If $B$ is an $(\mathcal{X}, \mathcal{Y})$-block of $G, x \in \mathcal{X}$ and $\sum_{\chi \in \operatorname{Irr}(G)} a_{\chi} \chi$ is a class function which vanishes identically on the $\mathcal{Y}$-section of $x$ in $G$, then $\sum_{\chi \in B} a_{\chi} \chi$ vanishes identically on the $\mathcal{Y}$-section of $x$ in $G$.

(3) $(\mathcal{X}, \mathcal{Y})$-blocks separate $\mathcal{Y}$-sections of $G$.

Proof: We have already proved (1) and (3) in the course of the proof of Theorem 2.1. For a (sketch) proof of (2) along these lines, note that if $b$ is any $(\mathcal{X}, \mathcal{Y})$-block of $C_{G}(x)$ which is a subset of $\beta=\beta(x, B)$, then the class function $\sum_{\chi \in \operatorname{Irr}(G)} a_{\chi} \chi^{(b)}$ vanishes identically on $x \mathcal{Y}(x)$, so that, by the Second Main Theorem property, $\sum_{\chi \in B} a_{\chi} \chi^{(b)}$ also vanishes identically on $x \mathcal{Y}(x)$. Hence $\sum_{\chi \in B} a_{\chi} \chi^{(\beta)}$ vanishes identically on $x \mathcal{Y}(x)$. But $\sum_{\chi \in B} a_{\chi} \chi^{(\beta)}$ agrees with $\sum_{\chi \in B} a_{\chi} \chi$ on $x \mathcal{Y}(x)$, again by the Second Main Theorem property.

\section{On $\ell$-Sections, $\ell$-Blocks and Brauer's Second Main Theorem}

Throughout this section, $G=S_{n}, \ell \geq 2$ is an integer and $\pi$ is the set $\pi(\ell)$ of primes dividing $\ell$. An $\ell$-cycle element (in a symmetric group) is an element with all non-trivial (disjoint) cycles of length divisible by $\ell$ and an $\ell$-regular element is an element with no cycle of length divisible by $\ell$. An $\ell$-singular element is an element with at least one cycle of length divisible by $\ell$. An $\ell$-element is an $\ell$-cycle element with each non-trivial cycle of length dividing a power of $\ell$. Moreover a $\pi$-regular element is an element whose order is not divisible by any prime in $\pi$.

We say that two elements $x$ and $y$ of $S_{n}$ are disjoint if $x$ fixes the points moved by $y$ and vice versa. If $x$ and $y$ are disjoint we write $x * y$ for the product to signify this. We may then also consider $y$ as a permutation of the fixed points of $x$ and vice versa. In particular when $x \in S_{k}$ and $y \in S_{m}$, it may be convenient to consider $x * y$ as an element of $S_{k+m}$. Clearly any element $z$ of $S_{n}$ may be factored uniquely into a product $x * y$ where $x$ is an $\ell$-cycle element and $y$ is $\ell$-regular. 
If we consider the factorization $z=x * y$ as above, we may factor the $\ell$-cycle element $x$ into a product $x=r s_{0}$ of commuting factors where $r$ is the $\pi$-part and $s_{0}$ is the $\pi^{\prime}$-part of $x$. Then $r$ is an $\ell$-element moving exactly the same points as $x$ and $s_{0}$ is $\ell$-regular. Moreover $r$ and $s_{0}$ are both disjoint from $y$. Thus $r, s_{0}, y$ are pairwise permutable, and $s=s_{0} * y$ is $\ell$-regular. Thus $x * y=r s$ and $z$ has a factorization of the form $r s$ where $r$ is an $\ell$-element, $s$ is $\ell$-regular and $r s=s r$. We call $x$ the $\ell$-cycle part of $z$, and $r$ the $\ell$-part of $z$.

Notice that if $w \in S_{n}$ commutes with $z$, then $w$ commutes with the $\ell$-cycle element $x$, hence also with $y, r, s_{0}$ and $s$.

We have shown:

Lemma 3.1. Each element $z \in S_{n}$ has unique factorizations

$$
z=x * y=r s=s r,
$$

where $x$ is an $\ell$-cycle element, $y$ is $\ell$-regular, $r$ (an $\ell$-element) is the $\pi$-part of $x$ and $s$ is $\ell$-regular. Any element commuting with $z$ commutes with each of $x, y, r, s$ (in particular, these elements all commute with each other).

Two elements of $S_{n}$ are said to belong to the same $\ell$-cycle section if their $\ell$-cycle parts are conjugate in $S_{n}$. Two elements of $S_{n}$ are said to be in the same $\ell$-section, if their $\ell$-parts are conjugate in $S_{n}$.

We remark that each $\ell$-section of $S_{n}$ is a union of $\ell$-cycle sections. However, the set of $\ell$-regular elements of $S_{n}$ is both an $\ell$-section and an $\ell$-cycle section and we denote it by $S_{n}^{(\ell-r e g)}$. We now turn to the definition of suitable blocks (in the sense of section 2) for $S_{n}$. For a given $\ell$, it turns out that there are two natural choices.

We let $\mathcal{X}$ be the set of $\ell$-elements of $S_{n}$. For each $r \in \mathcal{X}, C=C_{S_{n}}(r)$ has a factorization in the form $C=C_{0} \times C_{1}$, where $C_{1}$ is the pointwise stabilizer of the points moved by $r$ and $C_{0}$ is the pointwise stabilizer of the points fixed by $r$ (note for future reference that $\left.r \in C_{0}\right)$. We let $\mathcal{Y}(r)$ be the set of elements of the form $s_{0} * s_{1}$, where $s_{0}$ is a $\pi^{\prime}$-element of $C_{0}$ and $s_{1}$ is an $\ell$-regular element of $C_{1}$. We let $\mathcal{X}^{\prime}$ be the set of $\ell$-cycle elements in $S_{n}$. For each $x \in \mathcal{X}^{\prime}$ we let $\mathcal{Y}^{\prime}(x)$ be the set of $\ell$-regular elements which are disjoint from $x$. Then the $\mathcal{Y}$-sections of $\ell$-elements are exactly the $\ell$-sections of $S_{n}$ and the $\mathcal{Y}^{\prime}$-sections of $\ell$-cycle elements are exactly the $\ell$-cycle sections of $S_{n}$. With this notation, $\mathcal{Y}(1)=\mathcal{Y}^{\prime}(1)=S_{n}^{(\ell-r e g)}$. However the non-trivial sections may differ, even when an $\ell$-element in $\mathcal{X}$ is considered as an $\ell$-cycle element in $\mathcal{X}^{\prime}$.

Referring to Section 2 we see that the $(\mathcal{X}, \mathcal{Y})$-blocks and the $\left(\mathcal{X}^{\prime}, \mathcal{Y}^{\prime}\right)$-blocks of $S_{n}$ are identical. It is only when we pass to centralizers that the distinction between the two types of blocks becomes apparent.

Definition. We refer to an $(\mathcal{X}, \mathcal{Y})$-block of the centralizer of an $\ell$-element (possibly the identity) of $S_{n}$ as a linked $\ell$-block. Similarly $\left(\mathcal{X}^{\prime}, \mathcal{Y}^{\prime}\right)$-blocks of centralizers of $\ell$-cycle elements of $S_{n}$ are called cycle linked $\ell$-blocks. We refer to characters in the same linked (or cycle linked) $\ell$-block as being $(\ell$-)linked (or $(\ell-)$ cycle linked).

For each $r \in \mathcal{X}$, let $C_{S_{n}}(r)=C_{0} \times C_{1}$ be the factorization described above. Now $O_{\pi}\left(C_{0}\right)$ is easily seen to contain its centralizer in $C_{0}$ (we note that $C_{0}$ acts faithfully on the points moved by $r$, and is isomorphic to a direct product of groups of the form $\mathbb{Z}_{t \ell}$ ? $S_{m}$, where $t$ is an integer only divisible by primes in $\pi$ ). Hence $C_{0}$ has a unique $\pi$-block by Theorem 9 of [14]. Since $\pi$-blocks are characterized in [14] in terms of linking across the set of $\pi$-regular elements, it follows that a linked $\ell$-block 
of $C_{S_{n}}(r)$ is a set of irreducible characters of the form $\alpha_{0} \otimes \alpha_{1}$, where $\alpha_{0}$ ranges over all irreducible characters of $C_{0}$ and $\alpha_{1}$ ranges through the irreducible characters in a fixed (linked) $\ell$-block of $C_{1}$.

The irreducible characters and the conjugacy classes of $S_{n}$ are labelled canonically by the partitions of $n$. If $\lambda$ is a partition of $n$ then $\chi_{\lambda}$ denotes the irreducible character of $S_{n}$, labelled by $\lambda$.

We associate to $\lambda$ its $(\ell-)$ core $\gamma_{\lambda}$ and its $(\ell-)$ quotient $\beta_{\lambda}$. (See [6], Section 2.7.) The $\ell$-core is obtained from $\lambda$ by removing all $\ell$-hooks from $\lambda$. The number of $\ell-$ hooks to be removed from $\lambda$ to go to the core is called the $(\ell-)$ weight of $\lambda$ and denoted $w_{\lambda}$. The quotient $\beta_{\lambda}$ is an $\ell$-tuple of partitions

$$
\left(\beta_{0}, \beta_{1}, \ldots, \beta_{\ell-1}\right),
$$

whose cardinalities add up to $w_{\lambda}$. It is known that $\beta_{\lambda}$ and $\gamma_{\lambda}$ determine $\lambda$ uniquely. The quotient is also known as the "star diagram" in the work of G. de B. Robinson and Osima. We call an $\ell$-tuple of partitions whose cardinalities add up to $w$ simply an ( $\ell$-)quotient of $w$. The set of $\ell$-quotients of $w$ is denoted $\mathcal{K}(\ell, w)$ and the cardinality of this set is called $k(\ell, w)$. Thus

$$
k(\ell, w)=\sum_{w_{0}, w_{1}, \ldots, w_{\ell-1}} p\left(w_{0}\right) p\left(w_{1}\right) \ldots p\left(w_{\ell-1}\right),
$$

where the $w_{i}^{\prime}$ s are nonnegative integers satisfying $w_{0}+w_{1}+\ldots+w_{\ell-1}=w$ and $p(w)$ is the number of partitions of $w$.

By the core, $\ell$-core of an irreducible character $\chi$ we mean the $\ell$-core of $\lambda$, if $\chi=\chi_{\lambda}$.

Definition. Given a (possibly trivial) $\ell$-element $r$ of $S_{n}$, a combinatorial $\ell$-block of $C_{S_{n}}(r)\left(=C_{0} \times C_{1}\right.$ as usual) is a set of irreducible characters of the form $\alpha_{0} \otimes \alpha_{1}$, where $\alpha_{0}$ ranges over all irreducible characters of $C_{0}$ and $\alpha_{1}$ ranges through the irreducible characters of $C_{1}$ with a fixed $\ell$-core.

Remark. It may be asked whether linked $\ell$-blocks and combinatorial $\ell$-blocks of $S_{n}$ coincide. This is indeed true for every $\ell \geq 2$. It is fairly easy to show that a combinatorial $\ell$-block is a union of linked $\ell$-blocks (See Proposition 3.4). The proof of the converse is based on a result in Section 5, so we postpone it. (See Theorem $5.13)$.

Let us return to the $\ell$-cycle elements. If the cycle type of the $\ell$-cycle element $x$ is $\left(\ell r_{1}, . ., \ell r_{t}\right)$, (parts equal to 1 omitted), then we call $\rho=\left(r_{1}, . ., r_{t}\right)$ the $\ell$-type of $x$ (and of $x * y$, when $y$ is $\ell$-regular). Moreover if $|\rho|=v$, we call $v$ the $\ell$ weight of $x * y$. We include here the possibility that $\rho=0$, the empty partition. Thus the conjugacy classes of $\ell$-cycle elements of $S_{n}$ are parametrized canonically by partitions $\rho$ satisfying $\ell|\rho| \leq n$. We denote the $\ell$-cycle section consisting of elements of $\ell$-type $\rho$ by $S_{n}^{\rho}$. In particular, $S_{n}^{0}=S_{n}^{(\ell-r e g)}$ is the set of $\ell$-regular elements in $S_{n}$.

Once more, let $\chi_{\lambda}$ denote the irreducible character of $S_{n}$, labelled by the partition $\lambda$ of $n$. If $x$ is an $\ell$-cycle element of type $\rho$ as above and $y$ is an arbitrary element disjoint from $x$, then repeated use of the Murnaghan-Nakayama formula shows that

$$
\chi_{\lambda}(x * y)=\sum_{|\mu|=n-v \ell} m_{\lambda \mu}^{\rho} \chi_{\mu}(y)
$$


where the coefficients $m_{\lambda \mu}^{\rho}$ are integers, which we call Murnaghan-Nakayama coefficients or $M N$-coefficients for short.

The coefficient $m_{\lambda \mu}^{\rho}$ is clearly nonzero only if it is possible to go from $\lambda$ to $\mu$ by first removing an $\ell r_{1}$-hook from $\lambda$, then removing an $\ell r_{2}$-hook from the resulting partition and so on. Each sequence of such hook-removals defines a path $P$ in the lattice of partitions. More details are given later.

Lemma 3.2. If $m_{\lambda \mu}^{\rho} \neq 0$, then $\lambda$ and $\mu$ have the same $\ell$-core.

Proof: The lemma follows from the well-known fact that the removal of one hook of length $\ell r$ may also be accomplished by removing a sequence of $r$ hooks of length $\ell$. This fact is seen easily eg. using the $\ell$-abacus ([6], Section 2.7. See also Theorem (3.3) in [11].)

When $\rho$ is a partition of $v, v \ell \leq n$, and $\lambda, \lambda^{\prime}$ are partitions of $n$, we define

$$
g_{\lambda \lambda^{\prime}}^{\rho}=(1 / n !) \sum_{g \in S_{n}^{\rho}} \chi_{\lambda}(g) \chi_{\lambda^{\prime}}(g),
$$

the contribution of the $\ell$-cycle section of type $\rho$ to the inner product of the two irreducible characters. (If we take $\mathcal{C}=S_{n}^{\rho}$, this is in accordance with section 1) The orthogonality relations show that we have the equation

$$
\sum_{\rho} g_{\lambda \lambda^{\prime}}^{\rho}=\delta_{\lambda \lambda^{\prime}}
$$

The contributions may also be calculated as follows. Suppose that $x$ is an $\ell$-cycle element of type $\rho,|\rho|=v$. Then

$$
g_{\lambda \lambda^{\prime}}^{\rho}=(1 / n !) \sum_{y \in S_{n-v \ell}^{(\ell-r e g)}} \chi_{\lambda}(x * y) \chi_{\lambda^{\prime}}(x * y)
$$

We may then invoke the $M N$-coefficients (1) to get the following equation:

$$
g_{\lambda \lambda^{\prime}}^{\rho}=((n-v \ell) ! / n !) \sum_{\mu, \mu^{\prime}} m_{\lambda \mu}^{\rho} g_{\mu \mu^{\prime}}^{0} m_{\lambda^{\prime} \mu^{\prime}}^{\rho}
$$

Let $g_{\lambda \lambda^{\prime}}=g_{\lambda \lambda^{\prime}}^{0}$. Note that $g_{\lambda \lambda^{\prime}} \neq 0$ if and only if the corresponding characters $\chi_{\lambda}$ and $\chi_{\lambda^{\prime}}$ are directly linked in the sense of Section 1 , if $\mathcal{C}=S_{n}^{(\ell-r e g)}$.

Lemma 3.3. If $g_{\lambda \lambda^{\prime}}^{\rho} \neq 0$ then $\lambda$ and $\lambda^{\prime}$ have the same $\ell$-core.

Proof: We assume that $\lambda \neq \lambda^{\prime}$ and use induction on $n$. The result is obviously true for $n<\ell$. Assume first that $\rho \neq 0$. Using Lemma 3.2, formula (4) and the induction hypothesis we see that the statement is true in this case. If $\rho=0$, then by formula (2) there exists a $\rho^{\prime} \neq 0$, such that $g_{\lambda \lambda^{\prime}}^{\rho^{\prime}} \neq 0$. Then we are done by the previous case.

Proposition 3.4. If the irreducible characters $\chi_{\lambda}$ and $\chi_{\lambda^{\prime}}$ are $\ell$-linked or $\ell$-cycle linked, then $\lambda$ and $\lambda^{\prime}$ have the same $\ell$-core.

Proof: Follows from Lemma 3.3 and the definition of $\ell$-linking.

Remark. Our results show in fact that if two irreducible characters are linked via any $\ell$-section or any $\ell$-cycle section, then their partitions have the same $\ell$-core. 
Remark. If we arrange the irreducible characters in such a way that characters, whose labels have the same $\ell$-core are next to each other, then the matrices of $M N$-coefficients and of contributions have block forms, according to the possible cores, by Lemmas 3.2 and 3.3 .

The above shows that $\ell$-linked characters belong to the same combinatorial $\ell$ block. The converse statement is shown in Section 5. Until then we make a formal distinction between linked and combinatorial blocks.

We want now to establish the Second Main Theorem property for combinatorial $\ell$-blocks. We refer to the notation introduced in the beginning of this section.

Proposition 3.5. Let $\gamma$ be a fixed $\ell$-core. Let $\chi=\chi_{\lambda}$ be an irreducible character labelled by a partition with $\ell$-core $\gamma$. Choose $r \in \mathcal{X}$, and let $C_{S_{n}}(r)=C_{0} \times C_{1}$ as above. Then there exist complex numbers $d_{\chi}^{(r), \mu_{0}, \mu_{1}}$ such that for all $\pi$-regular $s_{0} \in C_{0}$ and all $s_{1} \in C_{1}$, we have:

$$
\chi\left(r s_{0} s_{1}\right)=\sum_{\mu_{0} \otimes \mu_{1}} d_{\chi, \mu_{0}, \mu_{1}}^{(r)} \mu_{0}\left(s_{0}\right) \mu_{1}\left(s_{1}\right),
$$

where $\mu_{0} \otimes \mu_{1}$ ranges over irreducible characters of $C_{0} \times C_{1}$ such that $\mu_{1}$ is labelled by a partition having $\ell$-core $\gamma$.

Proof: Before starting the proof let us note that there is no restriction at all on the irreducible characters $\mu_{0}$ of $C_{0}$ which may appear in the formula. The complex numbers $d_{\chi, \mu_{0}, \mu_{1}}^{(r)}$ should, of course, depend only on $r$ and not on $s_{0}$ or $s_{1}$.

Let us fix a choice of ( $\pi$-regular) $s_{0}$ for the moment. Then $x=r s_{0}$ is an $\ell$ cycle element. Applying formula (1) above to $x=r s_{0}, y=s_{1}$ we may find integers (Murnaghan-Nakayama coefficients), denoted here $c_{\mu_{1}}^{\left(r s_{0}\right)}$, such that for each $s_{1} \in C_{1}$, we may write

$$
\chi\left(r s_{0} s_{1}\right)=\sum_{\mu_{1}} c_{\mu_{1}}^{\left(r s_{0}\right)} \mu_{1}\left(s_{1}\right),
$$

where $\mu_{1}$ runs through irreducible characters of $C_{1}$ with $\ell$-core $\gamma$. The coefficients depend on $s_{0}$, but we will see that this is no obstacle to the desired conclusion.

Now let us allow $s_{0}$ to vary. We may certainly find (as usual, looking at representations of $C_{S_{n}}(r)$ on eigenspaces of $r$ ), complex numbers $d_{\chi, \mu_{0}, \mu_{1}}^{(r)}$ such that for all $s_{0}, s_{1}$ as above, we have :

$$
\chi\left(r s_{0} s_{1}\right)=\sum_{\mu_{0} \otimes \mu_{1}} d_{\chi, \mu_{0}, \mu_{1}}^{(r)} \mu_{0}\left(s_{0}\right) \mu_{1}\left(s_{1}\right),
$$

where $\mu_{0} \otimes \mu_{1}$ ranges over all irreducible characters of $C_{0} \times C_{1}$. We emphasize that the $d_{\chi, \mu_{0}, \mu_{1}}^{(r)}$ depend only on $r$. To be precise, we have

$$
d_{\chi, \mu_{0}, \mu_{1}}^{(r)}=\left\langle\operatorname{Res}_{C_{S_{n}}(r)}^{S_{n}}(\chi), \mu_{0} \otimes \mu_{1}\right\rangle \frac{\mu_{0}(r)}{\mu_{0}(1)} .
$$

But now, if we fix $s_{0}$ again, and define the class function $\theta^{\left(r, s_{0}\right)}$ of $C_{1}$ by setting $\theta^{\left(r, s_{0}\right)}\left(s_{1}\right)=\chi\left(r s_{0} s_{1}\right)$ for all $s_{1} \in C_{1}$, we have

$$
\theta^{\left(r, s_{0}\right)}=\sum_{\mu_{0} \otimes \mu_{1}} d_{\chi, \mu_{0}, \mu_{1}}^{(r)} \mu_{0}\left(s_{0}\right) \mu_{1},
$$


where $\mu_{0} \otimes \mu_{1}$ ranges over all irreducible characters of $C_{0} \times C_{1}$. On the other hand, as we saw above, we may also write

$$
\theta^{\left(r, s_{0}\right)}=\sum_{\mu_{1}} c_{\mu_{1}}^{\left(r, s_{0}\right)} \mu_{1}
$$

where $\mu_{1}$ runs through all irreducible characters of $C_{1}$ indexed by partitions with $\ell$ core $\gamma$. By the uniqueness of the expression of a class-function as a sum of irreducible characters, we conclude that $\sum_{\mu_{0} \in \operatorname{Irr}\left(C_{0}\right)} d_{\chi, \mu_{0}, \mu_{1}}^{(r)} \mu_{0}\left(s_{0}\right)=0$ unless that partition labelling $\mu_{1}$ has $\ell$-core $\gamma$.

Hence we may indeed delete irreducible characters $\mu_{0} \otimes \mu_{1}$ such that the partition labelling $\mu_{1}$ doesn't have $\ell$-core $\gamma$, from the expression

$$
\chi\left(r s_{0} s_{1}\right)=\sum_{\mu_{0} \otimes \mu_{1}} d_{\chi, \mu_{0}, \mu_{1}}^{(r)} \mu_{0}\left(s_{0}\right) \mu_{1}\left(s_{1}\right),
$$

as desired.

Theorem 3.6. Combinatorial $\ell$-blocks of $S_{n}$ satisfy the Second Main Theorem property.

Proof: To prove this result, it is necessary to show that for each $\ell$-element, $r$, of $S_{n}$ and each combinatorial $\ell$-block $b$ of (the usual) $C_{1}$ there is a unique combinatorial $\ell$-block $B$ of $S_{n}$ such that for some irreducible character $\chi$ of $B, \ell$-regular element $s_{1}$ of $C_{1}$ and $\pi$-regular element $s_{0}$ of (the usual) $C_{0}$ we have

$$
0 \neq \sum_{\alpha_{0} \in \operatorname{Irr}\left(C_{0}\right)} \sum_{\alpha_{1} \in b}\left\langle\operatorname{Res}_{C_{0} \times C_{1}}^{S_{n}}(\chi), \alpha_{0} \otimes \alpha_{1}\right\rangle \alpha_{0}\left(r s_{0}\right) \alpha_{1}\left(s_{1}\right) .
$$

Now let us choose a combinatorial $\ell$-block $b$ of $C_{1}$. This consists of all irreducible characters of $C_{1}$ which have a given $\ell$-core. We know from Proposition 3.4 that irreducible characters of $C_{1}$ which have different $\ell$-cores are orthogonal across the set of $\ell$-regular elements of $C_{1}$.

For $\chi$ an irreducible character of $S_{n}$, we set

$$
\chi^{(b)}=\sum_{\alpha_{0} \in \operatorname{Irr}\left(C_{0}\right)} \sum_{\alpha_{1} \in b}\left\langle\operatorname{Res}_{C_{0} \times C_{1}}^{S_{n}}(\chi), \alpha_{0} \otimes \alpha_{1}\right\rangle \alpha_{0} \otimes \alpha_{1} .
$$

Then for $\pi$-regular $s_{0} \in C_{0}$ and $\ell$-regular $s_{1} \in C_{1}$, we have

$$
\chi^{(b)}\left(r s_{0} s_{1}\right)=\sum_{\alpha_{0} \in \operatorname{Irr}\left(C_{0}\right)} \sum_{\alpha_{1} \in b}\left\langle\operatorname{Res}_{C_{0} \times C_{1}}^{S_{n}}(\chi), \alpha_{0} \otimes \alpha_{1}\right\rangle \frac{\alpha_{0}(r)}{\alpha_{0}(1)} \alpha_{0}\left(s_{0}\right) \alpha_{1}\left(s_{1}\right),
$$

and we have seen above that this is 0 unless $\chi$ has the same $\ell$-core as that defining $b$. In conclusion, we see that the unique choice of combinatorial $\ell$-block $B$ is that labelled by the same $\ell$-core as $b$.

Remarks: The observant reader may notice that we have verified one formulation of the Second Main Theorem property for combinatorial $\ell$-blocks, while the equivalent formulations of the Second Main Theorem property in section 2 were proved for blocks defined according to linking. In fact, since combinatorial $\ell$-blocks are unions of linked $\ell$-blocks, the necessary adaptations of the proofs from section 2 could be made in order to obtain analogues of the other formulations for combinatorial $\ell$-blocks. However, since (as we have remarked already), we will eventually see that linked and combinatorial $\ell$-blocks coincide, we content ourselves for the 
moment with proving one formulation of the Second Main Theorem property for combinatorial $\ell$-blocks.

It can be shown by a similar argument to that of the proof of Theorem 3.6 that combinatorial $\left(\mathcal{X}^{\prime}, \mathcal{Y}^{\prime}\right)$-blocks of $S_{n}$ (defined in the obvious fashion) also satisfy the Second Main Theorem property.

\section{ON $\ell$-PROJECTIVE CHARACTERS AND BASIC SETS}

As in the previous section $S_{n}^{(\ell-r e g)}$ is the set of $\ell$-regular elements of $S_{n}$. For any character (or class function) $\chi$ we write $\chi^{(\ell-r e g)}$ for $\chi_{n}^{S_{n}^{(\ell-r e g)}}$, as in section 1 . Moreover we call any $\mathbb{Z}$-basis of $\mathcal{R}\left(S_{n}^{(\ell-r e g)}\right)$ an $\left(\ell\right.$-) basic set for $S_{n}$, and we refer to the elements of $\mathcal{P}\left(S_{n}^{(\ell-r e g)}\right)$ as ( $\ell-$ projective (generalized) characters of $S_{n}$.

A partition of $n$ is called $\ell$-regular if no part is repeated $\ell$ or more times. It is called $\ell$-class regular if no part is divisible by $\ell$.

In this section we construct a series of $\ell$-projective characters, labelled by the $\ell$ regular partitions of $n$ for each symmetric group $S_{n}$. We also show that the $\chi_{\mu}^{(\ell-r e g)}$, where $\mu$ runs through the set of $\ell$-regular partitions of $n$, form a basic set. Ideas from the proof of Theorem 6.3.50 in [6] are used. In particular we need some concepts from Section 6.3 of [6].

Given a partition $\lambda$ of $n$ we define its $\ell$-residue diagram by filling in the residues of $j-i$ modulo $\ell$ in the Young diagram of $\lambda$. For example, if $\lambda=(6,2,2,1), \ell=4$ we get the diagram:

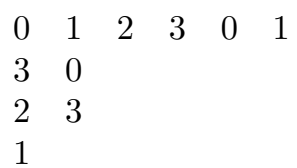

The $\ell$-content of $\lambda$ is defined as an integral vector $\left(c_{0}, c_{1}, \ldots, c_{\ell-1}\right)$, where $c_{i}$ is the number of nodes of residue $i$ in the $\ell$-residue diagram. In the above example it is $(3,3,2,3)$. By Theorem 2.7.41 in [6], two partitions of $n$ have the same $\ell$-core if and only if they have the same $\ell$-content. We also need the $\ell$-ladders. The $i$-th $\ell$-ladder is the straight line joining the point $(i, 1)$ to the point $\left(1,(i-1)(\ell-1)^{-1}+1\right)$. These ladders may contain nodes from the Young diagram of $\lambda$ and nodes on the same ladder have the same $\ell$-residue. If a ladder contains nodes from $\lambda$, we say that the ladder is "in $\lambda$ ". In the above example the fourth ladder is in $\lambda$ and contains two nodes of residue 1 in the positions $(4,1)$ and $(1,2)$. There is an $\ell$-regularization process associating to a partition $\lambda$ its regularized partition $\lambda^{R}$ obtained by moving all nodes on all ladders in $\lambda$ to the top positions on the ladder. The partitions $\lambda^{R}$ are $\ell$-regular and a partition $\lambda$ is $\ell$-regular if and only if $\lambda=\lambda^{R}$. The longest ladder of $\lambda$ is the $\ell$-ladder with the highest number in $\lambda$.

Example. $\lambda=(3,2,2,2,2,1), \ell=4$. Now $\lambda$ is not 4-regular, since the part 2 is repeated 4 times.

$\begin{array}{lll}0 & 1 & 2 \\ 3 & 0 & \\ 2 & 3 & \\ 1 & 2 & \\ 0 & 1 & \\ 3 & & \end{array}$


To 4-regularize this we need just to move the node of residue 1 in position $(5,2)$ to position $(2,3)$. Thus $\lambda^{R}=(3,3,2,2,1,1)$.

Theorem 4.1. For each $n$ there exists an integral matrix $D_{n, \ell}^{\prime}=\left(d_{\lambda \mu}^{\prime}\right)$ with nonnegative entries satisfying the following: The rows are indexed by partitions $\lambda$ of $n$ and its columns by $\ell$-regular partitions $\mu$ of $n$. Furthermore

(1) If $d_{\lambda \mu}^{\prime} \neq 0$ then $\mu$ dominates $\lambda^{R}$ and $\lambda$ and $\mu$ have the same $\ell$-core.

(2) If $\mu=\lambda^{R}$ then $d_{\lambda \mu}^{\prime}=1$.

(3) If the longest $\ell$-ladder in $\mu$ contains $k$ nodes of $\ell$-residue $r$, then $d_{\lambda \mu}^{\prime}=0$ unless a total of $k r$-nodes may be removed from $\lambda$.

(4) For all $\ell$-regular partitions $\mu$ the character $\phi_{\mu}^{\prime}$ defined by

$$
\phi_{\mu}^{\prime}=\sum_{|\lambda|=n} d_{\lambda \mu}^{\prime} \chi_{\lambda}
$$

is $\ell$-projective.

Proof: We use induction on $n$. For $n \leq \ell-1$ the result is trivial, since all characters are $\ell$-projective. Suppose the result has been proved up to and including $n-1$. Let $\mu$ be an $\ell$-regular partition of $n$. Assume that the longest ladder in $\mu$ contains $k$ nodes. These nodes are removable in $\mu$, since otherwise a longer ladder would be in $\mu$, and they all have the same $\ell$-residue $r$, say. Let $\mu^{\prime}$ be the partition of $n-k$ obtained from $\mu$ by removing these $k$ nodes. Then $\mu^{\prime}$ is again an $\ell$-regular partition. By the induction hypothesis there is an $\ell$-projective character $\phi_{\mu^{\prime}}^{\prime}$ of $S_{n-k}$, say

$$
\phi_{\mu^{\prime}}^{\prime}=\sum_{\left|\lambda^{\prime}\right|=n-k} d_{\lambda^{\prime} \mu^{\prime}}^{\prime} \chi_{\lambda^{\prime}}
$$

indexed by $\mu^{\prime}$. The coefficients $d_{\lambda^{\prime} \mu^{\prime}}$ satisfy conditions (1)-(3) above. Then the induced character $\phi^{*}=\operatorname{In} d_{S_{n-k}}^{S_{n}}\left(\phi_{\mu^{\prime}}^{\prime}\right)$ is also $\ell$-projective. By Proposition 3.4 the set of irreducible characters with a given core is a union of $\ell$-linked blocks. Therefore, by Lemma 1.1(1) the character $\phi_{\mu}^{*}$ obtained by removing all summands from $\phi^{*}$ where the partitions have an $\ell$-core different from the $\ell$-core of $\mu$ is again $\ell$-projective. We write

$$
\phi_{\mu}^{*}=\sum_{|\lambda|=n} d_{\lambda}^{\prime} \chi_{\lambda}
$$

where the coefficients by definition are non-negative. Let us note that $d_{\mu}^{\prime} \neq 0$ because $d_{\mu^{\prime} \mu^{\prime}}^{\prime}=1$. Thus $\phi_{\mu}^{*} \neq 0$. We want to show:

$\left(1^{*}\right)$ For all $\lambda$ we have $k ! \mid d_{\lambda}^{\prime}$.

$\left(2^{*}\right)$ If $d_{\lambda}^{\prime} \neq 0$ then $\mu$ dominates $\lambda^{R}$

$\left(3^{*}\right)$ If $\mu=\lambda^{R}$ then $d_{\lambda}^{\prime}=k$ !

$\left(4^{*}\right) d_{\lambda}^{\prime}=0$ unless a total of $k r$-nodes can be removed from $\lambda$.

Once this is proved we may divide $\phi_{\mu}^{*}$ by $k$ ! to obtain a new $\ell$-projective character $\phi_{\mu}^{\prime}$, whose coefficients $d_{\lambda \mu}^{\prime}$ satisfy the conditions of Theorem 4.1 and we are done.

Suppose $d_{\lambda}^{\prime} \neq 0$. Then there exists a partition $\lambda^{\prime}$ of $n-k$ such that $d_{\lambda^{\prime} \mu^{\prime}}^{\prime} \neq 0$ and $\left\langle\operatorname{Ind} d_{S_{n-k}}^{S_{n}}\left(\chi_{\lambda^{\prime}}\right), \chi_{\lambda}\right\rangle \neq 0$. By assumption $\lambda$ and $\mu$ have the same $\ell$-core, since $d_{\lambda}^{\prime} \neq 0$ and $\lambda^{\prime}$ and $\mu^{\prime}$ have the same $\ell$-core, since $d_{\lambda^{\prime} \mu^{\prime}}^{\prime} \neq 0$. By definition of $\mu^{\prime}$, the $\ell$-contents of $\mu$ and $\mu^{\prime}$ differ only by $k$ in residue $r$. Thus the same has to be true for the $\ell$-contents of $\lambda$ and $\lambda^{\prime}$. Therefore the branching theorem shows that $\left\langle\operatorname{Ind}_{S_{n-k}}^{S_{n}}\left(\chi_{\lambda^{\prime}}\right), \chi_{\lambda}\right\rangle=k$ !, if $k$-nodes of residue $r$ can be added to $\lambda^{\prime}$ to get $\lambda$ and is 
0 otherwise. This proves $\left(1^{*}\right)$ and $\left(4^{*}\right)$. Since $d_{\lambda^{\prime} \mu^{\prime}}^{\prime} \neq 0$ we have that $\lambda^{\prime} R$ dominates $\mu^{\prime}$, by the induction hypothesis. Since by the above $\lambda$ is obtained from $\lambda^{\prime}$ by adding $k$ nodes of residue $r$, the combinatorial lemma 6.3.54 in [6] shows that $\mu$ dominates $\lambda^{R}$. This shows $\left(2^{*}\right)$. Finally, suppose that $\mu=\lambda^{R}$. We have

$$
d_{\lambda}^{\prime}=\sum_{\lambda^{\prime}} d_{\lambda^{\prime} \mu^{\prime}}^{\prime}\left\langle\operatorname{In} d_{S_{n-k}}^{S_{n}}\left(\chi_{\lambda^{\prime}}\right), \chi_{\lambda}\right\rangle
$$

where the sum is on $\lambda^{\prime}$ with the same $\ell$-core as $\mu^{\prime}$. By the combinatorial lemma 6.3.55 in [6] there is a unique partition $\lambda^{\prime}$ such that $d_{\lambda^{\prime} \mu^{\prime}}^{\prime} \neq 0$ and here in fact $\lambda^{\prime} R=$ $\mu^{\prime}$. By the induction hypothesis $d_{\lambda^{\prime} \mu^{\prime}}^{\prime}=1$ and by the above $\left\langle\operatorname{In} d_{S_{n-k}}^{S_{n}}\left(\chi_{\lambda^{\prime}}\right), \chi_{\lambda}\right\rangle=k$ !. Thus $\left(3^{*}\right)$ is shown and Theorem 4.1 follows.

As in [6], Theorem 3.6.60, we now have that the matrix $D_{n, \ell}^{\prime}$ is lower unitriangular, when the partitions labelling the rows are arranged starting with the $\ell$-regular partitions in lexicographic order followed by the $\ell$-singular partitions. This arrangement can also be made using only the partitions with the same $\ell$-core and it shows that the rows and columns in $D_{n, \ell}^{\prime}$ may also be arranged unitriangularily block-by-block. The following argument may then be applied both to $D(n, \ell)^{\prime}$ and to the submatrix for an $\ell$-block $B$ :

The top rows corresponding to the $\ell$-regular partitions form a triangular matrix $T$ with 1's in the diagonal and 0's below the diagonal. Let $T^{\prime}$ be its inverse. If we multiply $D_{n, \ell}^{\prime}$ on the right by $T^{\prime}$ we get a matrix $D_{n, \ell}$ with the unit matrix as the rows corresponding to $\ell$-regular partitions. This means simply that we have replaced the $\ell$-projective characters $\phi_{\mu}^{\prime}$ by other $\ell$-projective characters $\phi_{\mu}$. The entries $d_{\lambda \mu}$ of $D_{n, \ell}$ still satisfy the properties (1),(2) and (4) of Theorem 4.1, because $D_{n, \ell}$ may be obtained from $D_{n, \ell}^{\prime}$ by systematic column operations subtracting only multiples of the $j$-th column from the $i$-th when $i<j$.

Proposition 4.2. The $\chi_{\mu}^{(\ell-r e g)}$, where $\mu$ ranges over the $\ell$-regular partitions of $n$, form a basic set. Indeed, for any irreducible character $\chi_{\lambda}$ of $S_{n}$ we have in the above notation

$$
\chi_{\lambda}^{(\ell-r e g)}=\sum_{\mu \ell-r e g u l a r} d_{\lambda \mu} \chi_{\mu}^{(\ell-r e g)} .
$$

The integers $d_{\lambda \mu}$ satisfy analogues of the properties (1), (3) and (4) of Theorem 4.1.

Proof: We first show that the characters $\phi_{\mu}$ described above form a $\mathbb{Z}$-basis for the space $\mathcal{P}\left(S_{n}^{(\ell-r e g)}\right)$ of $(\ell-)$ projective characters. The $\phi_{\mu}$ 's certainly form a $\mathbb{Q}$-basis for $\mathcal{P}\left(S_{n}^{(\ell-r e g)}\right)$, as they are linearly independent over $\mathbb{Q}$. Let $\psi \in \mathcal{P}\left(S_{n}^{(\ell-r e g)}\right)$. Choose $t \in \mathbb{N}$ minimal such that $t \psi=\sum_{\mu \text { regular }} a_{\mu} \phi_{\mu}, a_{\mu} \in \mathbb{Z}$. We get $t \psi=\sum_{\mu, \lambda} a_{\mu} d_{\lambda \mu} \chi_{\lambda}$. The coefficent $\sum_{\mu} a_{\mu} d_{\lambda \mu}$ to each $\chi_{\lambda}$ is divisible by $t$. When $\lambda$ is regular, say $\lambda=\mu^{\prime}$, this sum has only one summand $a_{\mu^{\prime}}$. Thus all $a_{\mu}$ are divisible by $t$. The minimality of $t$ then forces $t=1$, as desired.

Now for each irreducible character $\chi_{\lambda}$ consider the generalized character $\hat{\chi}_{\lambda}=$ $\chi_{\lambda}-\sum_{\mu} d_{\lambda \mu} \chi_{\mu}$. Then for $\mu^{\prime}$ regular

$$
\left\langle\hat{\chi}_{\lambda}, \phi_{\mu^{\prime}}\right\rangle=\left\langle\chi_{\lambda}, \phi_{\mu^{\prime}}\right\rangle-\sum_{\mu} d_{\lambda \mu}\left\langle\chi_{\mu}, \phi_{\mu^{\prime}}\right\rangle=0
$$


since $\left\langle\chi_{\mu}, \phi_{\mu^{\prime}}\right\rangle=d_{\mu \mu^{\prime}}=\delta_{\mu, \mu^{\prime}}$. Thus $\hat{\chi}_{\lambda}$ is orthogonal to each character in $\mathcal{P}\left(S_{n}^{(\ell-r e g)}\right)$ and therefore it is 0 on the set $S_{n}^{(\ell-r e g)}$ of $\ell$-regular elements, proving the proposition.

The above remarks show that there is also a block version of Proposition 4.2:

Corollary 4.3. Let the partition $\lambda$ have $\ell$-core $\gamma$. Then we have in the above notation

$$
\chi_{\lambda}^{(\ell-r e g)}=\sum_{\mu \ell-\text { regular with core } \gamma} d_{\lambda \mu} \chi_{\mu}^{(\ell-r e g)} .
$$

\section{Equivalence of $\ell$-BLOCKS}

In this section we consider primarily combinatorial $\ell$-blocks of $S_{n}$. Such a block $B=B_{\gamma}$ consists of all characters $\chi_{\lambda}$ of $S_{n}$ with a given $\ell$-core $\gamma$. (See section 3.) We refer to $\gamma$ as the core $\gamma(B)$ of $B$ and define the weight $w=w(B)$ of $B$ as the common $\ell$-weight of the partitions labelling the characters in $B$. Thus if $\chi_{\lambda} \in B$ then

$$
|\lambda|=w(B) \ell+|\gamma(B)| .
$$

A main result is that the Cartan matrices of $\ell$-blocks of the same weight $w$ have the same invariant factors. In fact it is a consequence of Theorem 5.9, that they are perfectly isometric (in the sense of Section 1) to the set of all irreducible characters of $\mathbb{Z}_{\ell}$ ? $S_{w}$, with respect to Osima's set of "regular conjugacy classes" as described below. This also allows us to prove that linked and combinatorial blocks as defined in Section 3 are the same.

To enumerate the number $k(B)$ of characters in an $\ell$-block $B$ of weight $w$, we need only to quote Theorem 2.7.30 in [6]. The partitions labelling characters in $B$ are distinguished by their $\ell$-quotients. The number of $\ell$-quotients of $w$ is $k(\ell, w)$ as described in Section 3. Thus

Proposition 5.1. Let $B$ be an $\ell$-block. Then $k(B)=k(\ell, w(B))$.

In Proposition 4.2, a basic set was exhibited using the irreducible characters of $S_{n}$ labelled by the $\ell$-regular partitions $\mu$ of $n$. We have unique integers $d_{\lambda \mu}$ for each partition $\lambda$ and each $\ell$-regular partition $\mu$ such that the following holds

$$
\chi_{\lambda}(y)=\sum_{\mu \ell-\text { regular }} d_{\lambda \mu} \chi_{\mu}(y)
$$

for all $\ell$-regular elements $y$ in $S_{n}$. It was also shown that if $d_{\lambda \mu} \neq 0$ then $\lambda$ and $\mu$ have the same $\ell$-core. Moreover $d_{\mu \mu}=1$ for each $\ell$-regular partition $\mu$. The "decomposition matrix" $\left(d_{\lambda \mu}\right)$ splits into blocks and when arranged properly the decomposition matrix of an $\ell$-block $B$ (which we will in the following refer to as $D(B)$ ), is lower unitriangular with rows (columns) indexed by the set of partitions ( $\ell$-regular partitions) of $n$ with core $\gamma(B)$. The number of such $\ell$-regular partitions of $n$ with core $\gamma(B)$ is then the $\mathbb{Z}$-rank of the block and is denoted $l(B)$.

Proposition 5.2. Let $B$ be an $\ell$-block. Then $l(B)=k(\ell-1, w(B))$.

Proof: We have to enumerate the $\ell$-regular partitions with given weight and core. Consider a partition $\lambda$ written "exponentially" as $\left(1^{m_{1}}, 2^{m_{2}}, \cdots\right)$. Decompose each multiplicity $m_{i}=n_{i} \ell+r_{i}$, where $0 \leq r_{i} \leq \ell-1$. Then $\lambda$ determines and is obviously uniquely determined by the pair $\lambda_{s}, \lambda_{r}$ of partitions defined by $\lambda_{s}=$ $\left(1^{n_{1}}, 2^{n_{2}}, \cdots\right), \lambda_{r}=\left(1^{r_{1}}, 2^{r_{2}}, \cdots\right)$. It is easily seen that $\lambda$ and $\lambda_{r}$ have the same 
$\ell$-core and that $|\lambda|=\left|\lambda_{s}\right| \ell+\left|\lambda_{r}\right|$. Thus the number $k(\ell, w)$ of partitions of weight $w$ with a given core $\gamma$ may be decomposed as

$$
k(\ell, w)=\sum_{v \geq 0} p(v) l(w-v \ell, \gamma),
$$

where $l(w-v \ell, \gamma)$ is the number of $\ell$-regular partitions of weight $w-v \ell$ with core $\gamma$. Since the left hand side of the above equation is independent of $\gamma$, it follows easily by induction on $w$, that $l(w, \gamma)=k(\ell-1, w)$.

The above gives us the sizes of the decomposition matrix $D(B)$ and the Cartan matrix $C(B)=D(B)^{t} D(B)$ of $B$ in terms of $w(B)$.

The $\ell$-class regular partitions (as defined above) label the $\ell$-regular conjugacy classes. The number of partitions of $n$ is as before denoted $p(n)$ and the number of $\ell$-regular partitions of $n$ is denoted $p^{*}(n)$. This also equals the number of $\ell$ class regular partitions of $n$. The proof of this, which goes back to Glaisher in the nineteenth century, involves generating functions and thus does not depend on $\ell$ being a prime. (See also Lemma 6.1.2 in [6]). Clearly the number of conjugacy classes of $S_{n}$ contained in the $\ell$-cycle section $S_{n}^{\rho}$ equals the number of $\ell$-regular classes of $S_{n-v \ell}$, ie. $p^{*}(n-v \ell)$ and this depends only on $|\rho|=v$ and not on $\rho$ itself. Therefore we get the following important formula connecting the numbers $p(n)$ and $p^{*}(n)$ :

$$
p(n)=\sum_{v \geq 0} p(v) p^{*}(n-v \ell) .
$$

In continuation of section 3 we study further the $M N$-coefficients $m_{\lambda \mu}^{\rho}$ for a fixed $\rho$. Recall that

$$
\chi_{\lambda}(x * y)=\sum_{|\nu|=n-v \ell} m_{\lambda \nu}^{\rho} \chi_{\nu}(y)
$$

if $x$ is an $\ell$-cycle element of type $\rho$.

Let $X_{n}$ denote the character table of $S_{n}$. If $\rho$ is a partition of $v$, then $X_{n}^{\rho}$ is the submatrix of $X_{n}$ including only the columns corresponding to conjugacy classes containing elements of the form $x * y$, where $x$ is an $\ell$-cycle element of type $\rho$. This is a $p(n) \times p(n-v \ell)$-matrix, where again $p(n)$ denotes the number of partitions of $n$. Then

$$
X_{n}^{\rho}=M_{n}^{\rho} X_{n-v \ell},
$$

where $M_{n}^{\rho}$ is the $p(n) \times p(n-v \ell)$-matrix of $M N$ - coefficients $m_{\lambda \nu}^{\rho}$. The column orthogonality relations for the irreducible characters of $S_{n}$ show that

$$
\left(X_{n}^{\rho}\right)^{t} X_{n}^{\rho}=\Delta_{n, \rho},
$$

where $\Delta_{n, \rho}$ is a diagonal matrix. The diagonal entry corresponding to the partition $\kappa$ of $n-v \ell$ is the integer $z_{\rho \kappa}$, defined as the centralizer order of an element $x * y$, where $x$ is an $\ell$-cycle element of type $\rho$ and $y$ is an element of type $\kappa$. When $\rho$ is 0 , we put $\Delta_{n}=\Delta_{n, \rho}$. Column orthogonality also shows that $X_{n-v \ell}^{t} X_{n-v \ell}=\Delta_{n-v \ell}$. Thus we get by an easy calculation the following

Lemma 5.3.

$$
\left(M_{n}^{\rho}\right)^{t} M_{n}^{\rho}=X_{n-v \ell}\left(\Delta_{n, \rho} / \Delta_{n-v \ell}\right) X_{n-v \ell}^{-1} .
$$


¿From Lemma 5.3 we may compute easily the determinant of

$$
N_{n}^{\rho}:=\left(M_{n}^{\rho}\right)^{t} M_{n}^{\rho}
$$

combinatorially as the quotient of the determinants of two diagonal matrices.

Corollary 5.4. Let $\kappa$ be a partition of $n-v \ell$. Then the column of $X_{n-v \ell}$ corresponding to the conjugacy class of type $\kappa$ is an eigenvector for $N_{n}^{\rho}$ with eigenvalue $z_{\rho \kappa} / z_{\kappa}$, (where $z_{\kappa}$ as usual is the centralizer order of an element of conjugacy type $\kappa$.

Corollary 5.5. Suppose that $V$ is any column of $X_{n}$ not occuring in the submatrix $X_{n}^{\rho}$. Then $\left(M_{n}^{\rho}\right)^{t} V=0$.

Proof: Column orthogonality shows that $V^{t} X_{n}^{\rho}=0$. Since $X_{n-v \ell}$ is invertible, the result follows.

We now proceed to define $u$-numbers as follows. Let $H=S_{n-v \ell}$. By (1) we have for all partitions $\nu$ of $n-v \ell$ and all $\ell$-regular elements $y \in H$ that

$$
\chi_{\nu}(y)=\sum_{v \ell-\text { regular }} d_{\nu v} \chi_{v}(y) .
$$

Then with $\rho$ as before and $v \ell$-regular we define the $u$-numbers by

$$
u_{\lambda v}^{\rho}=\sum_{\nu} m_{\lambda \nu}^{\rho} d_{\nu v}
$$

If $u_{\lambda v}^{\rho} \neq 0$ then $\lambda$ and $v$ have the same $\ell$-core. (As in [7] it is possible to give an explicit formula for the $u$-numbers.)

The $u$-numbers $u_{\lambda v}^{\rho}$ may for a fixed $\rho$ and $v$ be arranged as a column of length $p(n)$. Putting all such columns together we get a matrix $U_{n}$ called the $u$-matrix. Let us note that $U_{n}$ is a square $p(n)$-matrix. Indeed the columns $U_{n}$ are indexed by pairs of partitions $\rho, v$ satisfying $|\rho| \ell+|v|=n$ where $\rho$ is arbitrary and $v$ is $\ell$-regular. By (2) above we see that the number of columns in $U$ is $p(n)$.

The matrix $U_{n}$ may be decomposed in two ways. The first decomposition is according to the $\ell$-cycle sections (collect the columns with a fixed $\rho$ ). The second decomposition is according to the $\ell$-blocks of $S_{n}$. Suppose that $B$ is an $\ell$-block of weight $w$ with core $\gamma$. Then we collect all those rows and columns where the $\lambda$ 's and $v$ 's have $\ell$-core equal to $\gamma$ to get the $u$-matrix $U(B)$ of $B$. This makes sense since $u_{\lambda v}^{\rho}$ is nonzero only if $\lambda$ and $v$ have the same core. We see that within a single block we may also arrange the $u$-numbers according to the $\ell$-cycle sections. The $\ell$-cycle section of $\rho$ occurs in $U(B)$ if and only if $v \leq w$. We have that $U(B)$ is a square $k(B)$-matrix. The number of columns in $U(B)$ associated with the $\ell$-cycle section of $\rho$ is $p^{*}(n-v \ell)$ when $v \leq w$. The proof of Proposition 5.2 confirms that $U(B)$ is indeed a square matrix.

We proceed to prove orthogonality relations for the $u$-numbers. In the case where $\ell$ is a prime number, they were proved by Osima in [12], but his ideas generalize easily to our case. We prefer again to use matrices.

If $\rho$ is a partition of $v$, we let $X_{n}^{* \rho}$ be the submatrix of $X_{n}^{\rho}$ above containing the columns of the conjugacy classes $x * y$ where $x$ is an $\ell$-cycle element of $\ell$-type $\rho$ and $y$ is $\ell$-regular in $S_{n-v \ell}$. This is a $p(n) \times p^{*}(n-v \ell)$-matrix. We let $Y_{n}$ denote the $\ell$-regular character table of $S_{n}$. This is defined as the square $p^{*}(n)$-submatrix 
of $X_{n}$ containing the character values of the irreducible characters indexed by $\ell$ regular partitions on $\ell$-regular conjugacy classes. Moreover $D_{n}=\left(d_{\lambda \mu}\right)$ is the decomposition matrix, defined by (1). By (1) and (3) we have

$$
\begin{aligned}
X_{n}^{* \rho} & =M_{n}^{\rho} X_{n-v \ell}^{* 0} \\
X_{n-v \ell}^{* 0} & =D_{n-v \ell} Y_{n-v \ell} .
\end{aligned}
$$

Combining these we see that

$$
U_{n}^{\rho}=M_{n}^{\rho} D_{n-v \ell}
$$

is the part of $U_{n}$ belonging to the $\ell$-cycle section of $\rho$ and that

$$
X_{n}^{* \rho}=U_{n}^{\rho} Y_{n-v \ell}
$$

By column orthogonality we have that

$$
\left(X_{n}^{* \rho}\right)^{t} X_{n}^{* \rho}=\Delta_{n, \rho}^{*},
$$

where $\Delta_{n, \rho}^{*}$ is the submatrix of $\Delta_{n, \rho}$ corresponding to the $\ell$-class regular partitions of $n-v \ell$. It follows that

$$
\left(Y_{n-v \ell}\right)^{t}\left(U_{n}^{\rho}\right)^{t} U_{n}^{\rho} Y_{n-v \ell}=\Delta_{n, \rho}^{*} .
$$

Thus the $\ell$-regular character tables are nonsingular. Moreover, when $\kappa$ is $\ell$-class regular then the partitions $\ell \rho$ and $\kappa$ have no parts in common. Therefore we get the equation

$$
z_{\rho, \kappa}=\ell^{t} z_{\rho} z_{\kappa}=z_{\rho}^{*}(v \ell) z_{\kappa}
$$

where $t$ is the length (number of parts) of $\rho$ and $z_{\rho}^{*}$ is the order of a suitable centralizer. ¿From Corollary 5.4 we conclude that

$$
N_{n}^{\rho} X_{n-v \ell}^{* 0}=z_{\rho}^{*}(v \ell) X_{n-v \ell}^{* 0}
$$

Thus by (7) and (6)

$$
\left(U_{n}^{\rho}\right)^{t} U_{n}^{\rho} Y_{n-v \ell}=D_{n-v \ell}^{t} N_{n}^{\rho} X_{n-v \ell}^{* 0}=z_{\rho}^{*}(v \ell) D_{n-v \ell}^{t} X_{n-v \ell}^{* 0} .
$$

We use (7) again and then multiply the equation from the right by the inverse of $Y_{n-v \ell}$ to get

$$
\left(U_{n}^{\rho}\right)^{t} U_{n}^{\rho}=z_{\rho}^{*}(v \ell) C_{n-v \ell}
$$

where $C_{n-v \ell}$ is the Cartan matrix. If $\rho \neq \rho^{\prime}$ then $\left(X_{n}^{* \rho}\right)^{t} X_{n}^{* \rho^{\prime}}=0$. Since the $\ell$ regular character tables are nonsingular we get from $(8)$ that $\left(U_{n}^{\rho}\right)^{t} U_{n}^{\rho^{\prime}}=0$. Thus we have the desired orthogonality relations for the $u$-numbers:

Proposition 5.6. Let $\rho$ and $\rho^{\prime}$ be different partitions with $\ell|\rho| \leq n$ and $\ell\left|\rho^{\prime}\right| \leq n$. Then

$$
\begin{gathered}
\left(U_{n}^{\rho}\right)^{t} U_{n}^{\rho}=z_{\rho}^{*}(v \ell) C_{n-v \ell} \\
\left(U_{n}^{\rho}\right)^{t} U_{n}^{\rho^{\prime}}=0 .
\end{gathered}
$$

In the remainder of this section we fix an $\ell$-block $B$ of $S_{n}$ of weight $w=w(B)$ with core $\gamma(B)=\gamma$ and we assume that $\rho$ is a partition of $v \leq w$. We have seen that $M N$-coefficients, decomposition numbers and $u$-numbers respect $\ell$-blocks in the sense that if one of these numbers is non-zero, then the partitions have the same core. Thus each non-zero number is associated to a unique core and thus to a unique block of $S_{n}$. We therefore get obvious block versions of earlier formulae like

$$
U^{\rho}(B)=M^{\rho}(B) D(b)
$$


where $b$ is the block of $S_{n-v \ell}$ of weight $w-v$ with core $\gamma$. Moreover, the block version of Proposition 5.6 looks like this:

Proposition 5.7. Let $B$ be as above. Let $\rho$ and $\rho^{\prime}$ be different partitions with $|\rho| \leq w$ and $\left|\rho^{\prime}\right| \leq w$. Then

$$
\begin{gathered}
U^{\rho}(B)^{t} U^{\rho}(B)=z_{\rho}^{*}(v \ell) C(b) \\
U^{\rho}(B)^{t} U^{\rho^{\prime}}(B)=0,
\end{gathered}
$$

where $b$ is the $\ell$-block of $S_{n-|\rho| \ell}$ with core $\gamma$.

Consider the "extreme" cases $v=0$ and $v=w$. When $v=0$, then the $u$ numbers are exactly the decomposition numbers for $B$, eg. by $(7)_{B}$. We want to show that when $v=w$ then $u_{\lambda \gamma}^{\rho}=m_{\lambda \gamma}^{\rho}$ is closely related to the character value of an irreducible character of the wreath product $S(\ell, w)=\mathbb{Z}_{\ell}$ 乙 $S_{w}$. (Lemma 5.8.) Thus, in a way, the u-numbers give a link between the decomposition matrix for $B$ and the irreducible characters of $S(\ell, w)$.

We have to specify more precisely the $M N$-coefficients $m_{\lambda \nu}^{\rho}$. Let $\rho=\left(r_{1}, r_{2}, \ldots, r_{t}\right)$ and let $\mathcal{P}_{\lambda \nu}^{\rho}$ be the set of paths $P$ in the lattice of partitions, obtained by removing a series of hooks of length $\ell r_{1}, \ldots, \ell r_{t}$ to go from $\lambda$ to $\nu$. Each path $P$ has a sign $\sigma_{P}$, defined as $(-1)^{t(P)}, t(P)$ being the sum of the leg lengths of the hooks in $P$. Then obviously

$$
m_{\lambda \nu}^{\rho}=\sum_{P \in \mathcal{P}_{\lambda \nu}^{\rho}} \sigma_{P}
$$

A special case of this is important. If $\nu=\gamma_{\lambda}$, the $\ell$-core of $\lambda$ and $\rho=\left(1^{w(\lambda)}\right)$ then $\sigma_{P}$ is independent of the choice of $P$ in $\mathcal{P}_{\lambda \gamma}^{\rho}$. (See eg. [10], p. 62-63, for details.) This common value of $\sigma_{P}$ is then called the $\ell$-sign of $\lambda$ and denoted by $\sigma_{\lambda}$.

As has been mentioned above the partitions $\lambda$ with $\ell$-core $\gamma$ are distinguished by their $\ell$-quotients. Thus there is a canonical bijection Quot $_{B}$ between the set $\operatorname{Irr}(B)$ of irreducible characters in $B$ and the set $\mathcal{K}(\ell, w(B))$, mapping $\chi_{\lambda}$ onto the quotient $\beta_{\lambda}$.

Since the removal of an $\ell r$-hook in $\lambda$ is reflected by the removal of an $r$-hook in one of the partitions occurring in $\gamma_{\lambda}$ there is an obvious canonical bijection $P \rightarrow \tilde{P}$ between $\mathcal{P}_{\lambda \nu}^{\rho}$ and the set $\mathcal{P}_{\beta(\lambda) \beta(\nu)}^{\rho}$ of $r_{1}, r_{2}, . ., r_{t}$-hook paths between the quotients of $\lambda$ and $\nu$. The sign $\sigma_{\tilde{P}}$ is then $(-1)^{t(\tilde{P})}, t(\tilde{P})$ being the sum of the leg lengths of the hooks in $\tilde{P}$. We have the following fundamental sign relation for corresponding paths (G. de B. Robinson, Osima):

$$
\sigma_{\lambda} \sigma_{P}=\sigma_{\nu} \sigma_{\tilde{P}}
$$

Let $S(\ell, w)$ denote the wreath product $\mathbb{Z}_{\ell}\left\{S_{w}\right.$. It is shown by Osima that there exist bijections between $\mathcal{K}(\ell, w)$ and the sets $\operatorname{Irr}(S(\ell, w))$ and $C C l(S(\ell, w))$ of irreducible characters and conjugacy classes of $S(\ell, w)$ respectively. In the case of characters this bijection is quite well-known. (See eg. [6], Chapter 4.) Since it is going to play an important rôle later, we look closer at the conjugacy classes. The group $S(\ell, w)$ is a semi-direct product of a base subgroup $\mathbb{Z}_{\ell}^{w}=\mathbb{Z}_{\ell} \times \cdots \times \mathbb{Z}_{\ell}$ of order $\ell^{w}$ and a group $\tilde{S}_{w}$ isomorphic to $S_{w}$ operating on the base subgroup by place permutations. Two elements of $S(\ell, w)$ are called disjoint, if their $\tilde{S}_{w}$-factors are disjoint. Thus we get a disjoint factorization of any element of $S(\ell, w)$ into "cycle 
factors", according to the cycles of its $\tilde{S}_{w}$-factor. Such a cycle factor is characterized (up to conjugacy) by a pair $(r, s)$, where $r$ is the cycle length in $\tilde{S}_{w}$ and $s$ is a residue $\bmod \ell$. It is conjugate to an element of $\tilde{S}_{w}$ if and only if $s=0$. That $s=0$ is equivalent to the fact that the product of the nonzero entries from the base subgroup occurring in the cycle factor is 1 . The partitions in the label of a conjugacy class are obtained by collecting cycle factors with the same residue. In particular the parts of 0 -th partition $\alpha_{0}$ of a conjugacy class label $\alpha$ describe the lengths $r$ of those cycle factors which are conjugate to cycles inside $\tilde{S}_{w}$.

In this context Osima proved a generalization of the Murnaghan-Nakayama formula (MN-formula). For details we refer to [13], Section 3. The formula should be clear once we explain what "hooks" and "cycles" are in quotients. A hook in a quotient $\beta$ is simply defined as a hook in one of the partitions in $\beta$ and hook-removal is defined correspondingly. A cycle in a quotient is a part in the 0 -th partition in the quotient.

When $\ell=1$, the result reduces to the usual MN-formula for $S_{w}$. But in contrast to the $S_{w}$-case the MN-formula can only be applied to those conjugacy classes of $S(\ell, w)$, where the 0-th partition of the label is non-trivial. Let us call such a class singular of type $\rho$ if the 0 -th partition in its label is $\rho$. Otherwise the class is called regular. To avoid confusion it may be pointed out that for $S(\ell, w)$ the terms regular and singular do not relate to the $\ell$-structure of the elements, not even when $S(\ell, w)$ is embedded canonically in the symmetric group $S_{\ell w}$.

The number of regular classes in $S(\ell, w)$ is then $k(\ell-1, w)=l(B)$ and the number of singular classes is $k(B)-l(B)$ by Propositions 5.1 and 5.2. For character values on a singular class of type $\rho=\left(r_{1}, . ., r_{t}\right),|\rho|=v$, we apply the generalized MNformula repeatedly $t$ times. Suppose that $\psi_{\beta}$ is the irreducible character of $S(\ell, w)$ labelled by the quotient $\beta \in \mathcal{K}(\ell, w)$. We get then for a singular element $\tilde{x} * \tilde{y}$ of type $\rho$ ( $\tilde{y}$ regular $)$, that

$$
\psi_{\beta}(\tilde{x} * \tilde{y})=\sum_{\delta \in \mathcal{K}(\ell, w-v)} \tilde{m}_{\beta \delta}^{\rho} \psi_{\delta}(\tilde{y})
$$

analogous to (3) and

$$
\tilde{m}_{\beta \delta}^{\rho}=\sum_{\tilde{P} \in \mathcal{P}_{\beta, \delta}^{\rho}} \sigma_{\tilde{P}}
$$

analogous to $(10)$

We now connect the characters of $B$ and $S(\ell, w)$. From (10), (11) and (12) we get for $\chi_{\lambda} \in B$ and $|\rho|=v \leq w$

$$
\sigma_{\lambda} m_{\lambda \nu}^{\rho}=\tilde{m}_{\beta_{\lambda} \beta_{\nu}}^{\rho} \sigma_{\nu}
$$

In the extreme case $v=w$ we now have that up to signs the $u$-numbers for $B$ are character values in $S(\ell, w)$ :

Lemma 5.8. Assume $\chi_{\lambda} \in B,|\rho|=w$ and that $\gamma$ is the core of B. Let $x$ be an $\ell$-cycle element of type $\rho$. Then

$$
\sigma_{\lambda} \chi_{\lambda}(x)=\psi_{\beta_{\lambda}}(\tilde{x}) \chi_{\gamma}(1)
$$

where $\tilde{x}$ is in the $S(\ell, w)$-conjugacy class labelled by $(\rho, 0, \cdots, 0)$, (singular of type $\rho)$. In particular

$$
\sigma_{\lambda} u_{\lambda \gamma}^{\rho}=\psi_{\beta_{\lambda}}(\tilde{x})
$$


Proof: Due to the assumptions there is only one non-zero summand when we apply (1) to $\chi_{\lambda}(x)$, namely for $\nu=\gamma$. Since $\sigma_{\gamma}=1$ we get, using (14) and (12)

$$
\sigma_{\lambda} \chi_{\lambda}(x)=\sigma_{\lambda} m_{\lambda \gamma}^{\rho} \chi_{\gamma}(1)=\tilde{m}_{\beta_{\lambda}, 0}^{\rho} \chi_{\gamma}(1)=\psi_{\beta_{\lambda}}(\tilde{x}) \chi_{\gamma}(1)
$$

Since $m_{\lambda \gamma}^{\rho}=u_{\lambda \gamma}^{\rho}$ by $(7)_{B}$ we are done.

Theorem 5.9. Let $D(B)$ be the decomposition matrix of $B$ and $Z_{w}^{0}$ the submatrix of the character table of $S(\ell, w)$ consisting of the columns of regular conjugacy classes in the sense defined above. These are both $k(B) \times l(B)$-matrices. Arrange the rows in these matrices such that the row in $D(B)$ corresponding to $\chi_{\lambda} \in B$ and the row corresponding to $\psi_{\beta_{\lambda}}$ in $Z_{w}^{0}$ have the same number. Let $\sigma(B)$ be the diagonal matrix with entries $\sigma_{\lambda}, \chi_{\lambda} \in B$. Then there exists a non-singular complex matrix $S(B)$ such that

$$
\sigma(B) D(B)=Z_{w}^{0} S(B) .
$$

Proof: We use induction on $w=w(B)$. For $w=0, D(B)$ and $Z_{w}^{0}$ are both the $1 \times 1$ unit matrix. Let $w=1$. Let $x$ be an $\ell$-cycle. By Lemma 5.8 we see that the column $\left(\sigma_{\lambda} u_{\lambda \gamma}^{1}\right), \chi_{\lambda} \in B$ equals the column $Z_{w}^{1}=\left(\psi_{\beta_{\lambda}}(\tilde{x})\right)$. By orthogonality of $u$-numbers (Proposition 5.7) we see that the columns of $\sigma(B) D(B)$ are orthogonal to the column $Z_{w}^{1}$. On the other hand the column orthogonality for $\operatorname{Irr}(S(\ell, w))$ shows that the columns of $Z_{w}^{0}$ form a basis for the space of columns orthogonal to $Z_{w}^{1}$. Thus our result is true in this case, too.

In the general case we note that the matrix form of (14) may be written as

$$
\sigma(B) M^{\rho}(B)=\tilde{M}_{w}^{\rho} \sigma(b),
$$

where $b$ is the block of weight $w-v$ with core $\gamma$. Applying the induction hypothesis to $b$ we see that when $\rho \neq 0$, then by $(7)_{B}$ there exists an invertible complex $l(b) \times l(b)$-matrix $S(b)$ such that

$$
\sigma(B) U^{\rho}(B)=\sigma(B) M^{\rho}(B) D(b)=\tilde{M}_{w}^{\rho} \sigma(b) D(b)=\tilde{M}_{w}^{\rho} Z_{w-v}^{0} S(b)=Z_{w}^{\rho} S(b),
$$

where $Z_{w}^{\rho}$ is the submatrix of the character table of $S(\ell, w)$ consisting of columns belonging to singular classes of type $\rho$. The last equality follows from (12). By orthogonality of $u$-numbers (Proposition 5.6) we see that the columns of $\sigma(B) D(B)$ are orthogonal to all columns in $Z_{w}^{\rho}$ for all $\rho \neq 0$. On the other hand the column orthogonality for $\operatorname{Irr}(S(\ell, w))$ shows that the columns of $Z_{w}^{0}$ form a basis for the space of columns orthogonal to all columns of the $Z_{w}^{\rho}$ 's. Thus the columns of $\sigma(B) D(B)$ are complex linear combinations of the columns in $Z_{w}^{0}$, as desired.

Theorem 5.10. Let $B$ and $B^{\prime}$ be $\ell$-blocks of weight $w$. There exists an integral invertible matrix $S$, such that

$$
\sigma(B) D(B)=\sigma\left(B^{\prime}\right) D\left(B^{\prime}\right) S
$$

and

$$
C(B)=S^{t} C\left(B^{\prime}\right) S,
$$

where $D(B), D\left(B^{\prime}\right)$ are the respective decomposition matrices and $C(B), C\left(B^{\prime}\right)$ are the respective Cartan matrices for $B$ and $B^{\prime}$. In particular $C(B)$ and $C\left(B^{\prime}\right)$ have the same invariant factors and the same determinant.

Proof: Let us arrange the rows in $D\left(B^{\prime}\right)$ such that the characters labelled by $\ell$ regular partitions (ordered lexicographically) are the first. Thus by (5) the top $l\left(B^{\prime}\right)$ rows of $D\left(B^{\prime}\right)$ form a unit matrix! We apply Theorem 5.9 to $B$ and $B^{\prime}$ to see that 
there exists an invertible complex matrix $S$, such that $\sigma(B) D(B)=\sigma\left(B^{\prime}\right) D\left(B^{\prime}\right) S$. However by the choice of the ordering of the rows in $D\left(B^{\prime}\right)$ we see that $S$ coincides with the first $l(B)=l\left(B^{\prime}\right)$ rows of $D(B)$. Thus $S$ is indeed integral.

It should be remarked that a choice of the ordering of the rows in one of the decomposition matrices in Theorem 5.10 also forces an ordering of the rows in the other. The orderings must be such that the partitions labelling the $i$-th row in $D(B)$ and $D\left(B^{\prime}\right)$ should have the same $\ell$-quotient. Examples show that whether a partition is $\ell$-regular or not does not only depend on the $\ell$-quotient but also on the core.

It turns out that theorem 5.9 allows us to prove that characters with the same core are linked. Using the notation of this theorem we get by an easy calculation that

$$
\sigma(B) D(B) C(B)^{-1} D(B)^{t} \sigma(B)=Z_{w}^{0}\left[{\overline{Z_{w}^{0}}}^{t} Z_{w}^{0}\right]^{-1}{\overline{Z_{w}^{0}}}^{t}
$$

This yields a perfect isometry in the sense of Section 1 . Indeed $\Gamma(\mathcal{C}, B)=$ $D(B) C(B)^{-1} D(B)^{t}$ is the matrix of contributions for the set of irreducible characters in $B$ with respect to $\mathcal{C}=S_{n}^{(\ell-r e g)}$, the union of the conjugacy classes of $\ell$-regular elements in $S_{n}$. Let us recall that the $\mathcal{C}$-blocks in the sense of Section 1 are just the linked $\ell$-blocks of $S_{n}$, and that by Proposition 3.4 the combinatorial $\ell$-block $B$ is a union of linked $\ell$-blocks.

Also $\Gamma\left(\mathcal{D}, B^{\prime}\right)=Z_{w}^{0}\left[{\overline{Z_{w}^{0}}}^{t} Z_{w}^{0}\right]^{-1} \bar{Z}_{w}^{t}$ is the matrix of contributions for the set $B^{\prime}=$ $\operatorname{Irr}(S(\ell, w))$ of all irreducible characters of $S(\ell, w)$ with respect to $\mathcal{D}=S(\ell, w)_{\text {reg }}$, the union of the regular conjugacy classes (in the above sense) in $S(\ell, w)$ (in what follows, we will sometimes abbreviate this just to reg for ease of notation).

Let us mention that $\mathcal{C}$ and $\mathcal{D}$ are both closed in the sense of section 1 . For $\mathcal{C}$ this is trivially true. For $\mathcal{D}$ it follows from the fact that no cycle factor of a regular element in $S(\ell, w)$ is conjugate to an element of $\tilde{S}_{w}$. If two elements of $S(\ell, w)$ generate the same cyclic subgroup, then each of their cycle factors have to generate the same cyclic subgroup (considered inside $S_{\ell w}$ ). Also the cycles in the $\tilde{S_{w}}$-parts of the elements generate the same cyclic subgroups of $\tilde{S_{w}}$. We then use the fact that a cycle element of type $(r, s)$ as above is a product of cycles of length $r \cdot \ell /(\ell, s)$, when considered as an element of $S_{\ell w}$.

Proposition 5.11. In the above notation $B$ and $B^{\prime}$ are perfectly isometric.

This implies also that if the latter matrix $\Gamma\left(\mathcal{D}, B^{\prime}\right)$ is indecomposable in the Frobenius-Perron sense (ie, there is no relabelling of rows and columns so that the matrix has a proper decomposition into block form), then so is the former matrix $\Gamma(\mathcal{C}, B)$. In other words, if all characters of $S(\ell, w)$ are linked across regular elements, then all irreducible characters of $B$ are linked across $\ell$-regular elements of $S_{n}$. Thus, in that case, all irreducible characters in $B$ would be in the same linked block.

Theorem 5.12. Every irreducible character of $S(\ell, w)$ is directly linked (across regular elements) to the trivial character.

Proof: For characters $\alpha$ and $\beta$ of $S(\ell, w)$, we let (as usual) $\langle\alpha, \beta\rangle_{\text {reg }}$ denote the truncation to regular elements of the usual inner product of $\alpha$ and $\beta$.

We want to calculate $\langle\chi, 1\rangle_{r e g}$ for $\chi$ an irreducible character of $S(\ell, w)$. We first consider the case that $\chi$ lies over an $S_{w}$-stable linear character $\lambda^{\prime}$ of the base group $\mathbb{Z}_{\ell}^{w}$. (In what follows $\mathbb{Z}_{\ell}$ is considered as a multiplicative group.) Notice that in 
that case, $\chi$ may be considered a character of $\mathbb{Z}_{d} \times S_{w}$ for some divisor $d$ of $\ell$, and that, as such, $\chi$ restricts irreducibly to the $S_{w}$ factor.

We note also in this case that $\lambda^{\prime}$ has the form $\lambda \otimes \ldots \otimes \lambda$ ( $w$ factors), where $\lambda$ is a linear character (of the order $d$ mentioned above) of the group $\mathbb{Z}_{\ell}$.

Let us now observe that for $\sigma$ in the complement $\tilde{S}_{w}$ to the base group, and any $w$-tuple $\left(a_{1}, \ldots, a_{w}\right)$ in $T$, we have $\chi\left(\left(a_{1}, \ldots, a_{w}\right) \sigma\right)=\lambda\left(a_{1} a_{2} \ldots a_{w}\right) \chi(\sigma)$.

Let's calculate the contribution to $\ell^{w} w !\langle\chi, 1\rangle_{\text {reg }}$ from the regular elements in the coset $T \sigma$. We have seen above that if $\sigma$ has disjoint cycle pattern $\left(r_{1}, \ldots r_{s}\right)$, an element $\left(a_{1}, a_{2}, \ldots, a_{w}\right) \sigma$ will be regular as long as, for each $i$, the $a$ 's appearing in positions labelled by the $r_{i}$ cycle have product different from 1 . We refer to such a $w$-tuple as permissible. It is routine to verify that

$$
\sum \lambda\left(a_{1} a_{2} \ldots a_{w}\right)=(-1)^{c(\sigma)} \ell^{w-c(\sigma)}
$$

if $\lambda$ is non-trivial, and is $(\ell-1)^{c(\sigma)} \ell^{w-c(\sigma)}$ if $\lambda$ is trivial, where the sum is taken over permissible $w$-tuples, and $c(\sigma)$ denotes the number of cycles of $\sigma$. To see this, note that there are $(\ell-1)^{c(\sigma)} \ell^{w-c(\sigma)}$ permissible $w$-tuples. Thinking one cycle at a time, note that for a given $t$-cycle of $\sigma$ there are $\ell^{t-1}(\ell-1)$ permissible $t$-tuples of elements of $\mathbb{Z}_{\ell}$ associated to this cycle (the product of the $t$-tuple must be a nonidentity element of $\mathbb{Z}_{\ell}$ ). In this special case, each non-identity element of $\mathbb{Z}_{\ell}$ occurs $\ell^{t-1}$ times as the product of a permissible $t$-tuple. Evaluating $\lambda$ on the product of each permissible $t$-tuple and adding the results gives $-\ell^{t-1}$ if $\lambda$ is non-trivial, $(\ell-1) \ell^{t-1}$ if $\lambda$ is trivial.

Hence we see that

$$
\ell^{w} w !\langle\chi, 1\rangle_{r e g}=\sum_{\sigma \in S_{w}} \chi(\sigma)\left(\delta_{\lambda, 1} \ell-1\right)^{c(\sigma)} \ell^{w-c(\sigma)} .
$$

In particular,

$$
\ell^{w} w !\langle\chi, 1\rangle_{\text {reg }} \equiv(-1)^{w} \chi(1)(\bmod \ell) .
$$

More precisely, since $\left[S_{w}: C_{S_{w}}(\sigma)\right] \chi(\sigma) / \chi(1)$ is an algebraic integer for each $\sigma \in S_{w}$, we deduce that

$$
\frac{\ell^{w} w !\langle\chi, 1\rangle_{\text {reg }}}{\chi(1)}
$$

is an integer congruent to $(-1)^{w}(\bmod \ell)$. In particular, it is not zero, and $\chi$ is linked across regular elements to the trivial character.

If $\chi$ does not lie over a stable linear character of the base group $T$, then $\chi$ is induced from a "Young subgroup" of the form $\mathbb{Z}_{\ell}^{w} \tilde{S}_{\lambda}$. Notice that regular elements of $S(\ell, w)$ remain regular in this Young subgroup (which is a direct product of smaller wreath products each with a base group which is a direct product of copies of $\mathbb{Z}_{\ell}$ ).

The character $\mu$ which induces to $\chi$ decomposes according to the direct factors of the above Young subgroup. We may suppose by induction that each of the factors of $\mu$ is directly linked across regular elements of the relevant factor of the Young subgroup. Hence $\mu$ is directly linked to the trivial character across regular elements of the whole Young subgroup. Frobenius reciprocity then tells us that $\chi$ is directly linked to the trivial character across regular elements. More precisely, an inductive argument tells us that

$$
\frac{\ell^{w} w !\langle\chi, 1\rangle_{r e g}}{\chi(1)}
$$


is an integer congruent to $(-1)^{w}(\bmod \ell)$ in this case too.

By the above remarks we have also shown

Theorem 5.13. The concepts of linked and combinatorial $\ell-$ blocks of $S_{n}$ are identical.

Let us remark that Theorem 5.13 is really the $\ell$-analogue of the Nakayama conjecture for symmetric groups, ([6], 6.2.21). In the case where $\ell$ is a prime, the theorem appears to provide yet another new proof of the Nakayama conjecture. Theorem 5.13, together with Corollary 2.2 and Proposition 3.6, also completes the proof that linked $\ell$-blocks of $S_{n}$ satisfy all the equivalent formulations of the Second Main Theorem property.

In the next section, we will study the invariant factors and determinants of Cartan matrices more closely.

\section{INVARIANT FACTORS}

The starting point here is that if we combine Proposition 1.4 and Proposition 5.11 we get:

Theorem 6.1. The invariant factors of the Cartan matrix $C(B)$ of an $\ell$-block $B$ of $S_{n}$ of weight $w$ are equal to the invariant factors of the Cartan matrix of $S(\ell, w)$ with respect to regular classes.

Note that, in particular, this provides an alternative proof of Theorem 5.10, since the latter Cartan matrix is uniquely specified by $\ell$ and $w$. The above equality of invariants arises from the fact that in the notation of Section 1 the Abelian groups $\operatorname{Cart}\left(S_{n}^{(\ell-r e g)}, B\right)$ and $\operatorname{Cart}\left(S(\ell, w)_{\text {reg }}\right)=\operatorname{Cart}(\ell, w)$ are isomorphic. In particular, these groups certainly have the same exponent. The results of Donkin [4] show that this exponent is a $\pi$-number, where $\pi=\pi(\ell)$ is the set of primes dividing $\ell$. Every positive integer $m$ factors uniquely as $m=m_{\pi} m_{\pi^{\prime}}$ where every prime factor of $m_{\pi}$ belongs to $\pi$ and no prime factor of $m_{\pi^{\prime}}$ is contained in $\pi$.

Theorem 6.2. The exponent of $\operatorname{Cart}(\ell, w)$ is $\ell^{w} w !_{\pi}$.

Proof: From the proof of Theorem 5.12, we see that $\left\langle\ell^{w} w ! 1, \chi\right\rangle_{\text {reg }}$ is integral for every irreducible character $\chi$ of $S(\ell, w)$, so that $\ell^{w} w !_{\pi} 1^{r e g}$ is a generalized character (using the fact that $\operatorname{Cart}(\ell, w)$ is a $\pi$-group). On the other hand, the proof of Theorem 5.12 also shows that

$$
\left\langle\ell^{w} w ! 1,1\right\rangle_{r e g} \equiv(-1)^{w}(\bmod \ell),
$$

so it easily follows that there is no prime $p \in \pi$ such that $\frac{\ell^{w} w !}{p} 1^{\text {reg }}$ is a generalized character, giving the result.

Corollary 6.3. The exponent of $\operatorname{Cart}\left(S_{n}^{(\ell-r e g)}\right)$ is $\ell^{\left.\ell^{\frac{n}{\ell}}\right\rfloor}\left\lfloor\frac{n}{\ell}\right\rfloor !_{\pi}$. This is the also the largest invariant factor of an $\ell$-Cartan matrix $C_{n}$ of $S_{n}$.

Remark. The two above results reflect the potential usefulness of Theorem 6.1. It may be easier to do calculations within $S(\ell, w)$ and to work with contributions (with respect to the set of regular elements) there. This was also illustrated in the proof of Theorem 5.12. The authors have been able to compute a generating function for the the entries of the matrix of "contributions" with respect to a $\mathbb{Z}$-basis for the 
character ring of $S(\ell, w)$ consisting of certain monomial characters. These entries are polynomials in $\ell$. The change of basis yields a new matrix of "contributions" which has exactly the same invariant factors as the original matrix of contributions. We intend to return to this at a later time.

Clearly the group $\operatorname{Cart}\left(S_{n}^{(\ell-r e g)}\right)$ has an order equal to the determinant of the Cartan matrix $C_{n}$. Below we give an explicit formula for this determinant, based on [3] and [1]. In addition we want here to present a conjecture about the structure of $\operatorname{Cart}\left(S_{n}^{(\ell-r e g)}\right)$, which we abbreviate $\operatorname{Cart}_{n}$. This will of course give the right determinant, and it is also supported by numerous explicit examples. When $\ell$ is a prime number, this conjecture is known to be true. Our inspiration has in fact been the prime case and Theorem 6.2.

In the prime case $\ell=p$ it is known that the invariant factors of the Cartan matrix are exactly the orders of the $p$-defect groups of $p$-regular conjugacy classes. This defect group is a direct product of the $p$-Sylow subgoups of wreath products $\mathbb{Z}_{m} 2 S_{a}$. Thus if a class regular partition is written exponentially $\left(1^{a_{1}}, \cdots, m^{a_{m}}, \cdots\right)$, then each "block" $m^{a_{m}}$ satisfying $a_{m} \geq p$ gives a contribution to the Cartan determinant, which is just the order of the $p$-Sylow subgroup of $S_{a_{m}}$.

In the general case there are obviously no defect groups of conjugacy classes or blocks. (In the case where $\ell$ is a power of the prime $p$, the group $\mathbb{Z}_{\ell} \ell P, P$ a $p$-Sylow subgroup of $S_{w}$, may in some sense be viewed as an "defect group" of an $\ell$-block of weight $w$.). In any case Corollary 6.3 suggests what the " $\ell$-defect" of $\left(1^{n}\right)$ should be. Numerous examples seem to indicate that in the composite case the contribution to the Cartan determinant of $\left(m^{a}\right)$, where $\ell \nmid m$, should not only depend on the multiplicity $a$, but also on the part $m$.

We use the notation $\lambda \vdash n\left(\lambda \vdash_{\ell} n\right)$ to signify that $\lambda$ is a ( $\ell$-class regular) partition of $n$. If $m$ is a positive integer, we define $\ell_{m}=\ell /(\ell, m)$ and $\pi_{m}$ as the set of primes dividing $\ell_{m}$. If $a$ is also a positive integer we set

$$
r_{\ell}(m, a)=\ell_{m}^{\lfloor a / \ell\rfloor} \cdot\lfloor a / \ell\rfloor !_{\pi_{m}} .
$$

Let the $\lambda \vdash_{\ell} n$ be written exponentially $\lambda=\left(1^{a_{1}(\lambda)}, 2^{a_{2}(\lambda)}, \cdots\right)$. We define

$$
r_{\ell}(\lambda)=\prod_{m} r_{\ell}\left(m, a_{m}(\lambda)\right) .
$$

Conjecture 6.4. The abelian group Cart $_{n}$ is a direct product of cyclic groups of order $r_{\ell}(\lambda)$, where $\lambda$ runs through the set of $\ell$-class regular partitions of $n$. In particular, the determinant of an $\ell$-Cartan matrix of $S_{n}$ is

$$
\operatorname{det}\left(C_{n}\right)=\prod_{\lambda \vdash \ell n} r_{\ell}(\lambda) .
$$

The invariant factors of the $\ell$-Cartan matrices of $S_{n}$ need not be powers of $\ell$. When $n<2 \ell$ it is quite easy to see that only 1 and $\ell$ occur as invariant factors, in accordance with our conjecture. But when $n \geq 2 \ell$, invariant factors occur which are not powers of $\ell$. We present three example. Exponents denote multiplicities of invariant factors.

Example 6.5. $n=8, \ell=4$ : The (principal) $\ell$-block $B$ of weight 2 has $k(B)=14$ and $l(B)=9$. The Cartan matrix $C(B)$ is a $9 \times 9$ matrix with invariant factors: $32,4^{2}, 2,1^{5}$. 
Example 6.6. $n=18, \ell=6$ : The invariant factors of $C_{n}$ are: $1296,72^{9}, 18,6^{55}, 3^{8}, 1^{223}$.

The $r_{\ell}(\lambda)$ 's for 6 -regular partitions are: $1296,72^{9}, 18,6^{54}, 3^{9}, 2,1^{222}$.

The determinant of $C_{n}$ and the product of the $r_{\ell}(\lambda)$ 's equal $2^{87} \cdot 3^{87}$. The number 87 may be calculated by Proposition 6.11 below.

Example 6.7. $n=24, \ell=6$ : The invariant factors of $C_{n}$ are: $31104,1296^{9}, 216,72^{54}, 36,18^{9}, 6^{231}, 3^{47}, 1^{760}$.

The $r_{\ell}(\lambda)$ 's for 6 -regular partitions are:

$31104,1296^{9}, 216,72^{54}, 18^{9}, 12,9,6^{222}, 3^{55}, 2^{9}, 1^{751}$

The determinant of $C_{n}$ and the product of the $r_{\ell}(\lambda)$ 's equal $2^{450} \cdot 3^{450}$. Again the number 450 may be calculated by Proposition 6.11 below.

It should be stressed that although in the examples there is a deviation between the lists of invariant factors and the $r_{\ell}$ 's, the examples are still in accordance with our conjecture.

It may seem slightly surprising that the divisors $\ell_{m}$ of $\ell$ should occur, especially since, as we shall soon see, the overall product of the $r_{\ell}(\lambda)$ 's should be a power of $\ell$. Indeed in support of our conjecture it is possible to show that

$$
\operatorname{det}\left(C_{n}\right)=\prod_{\lambda \vdash_{\ell} n} r_{\ell}(\lambda)
$$

and that $\operatorname{det}\left(C_{n}\right)$ is a specifically given power of $\ell$ which may be described by a simple combinatorial formula. (See Proposition 6.11.). Our proof is based on [4], [3] and [1]. A conjecture of A. Mathas states that the determinant of the Cartan matrix of an Iwahori-Hecke algebra of $S_{n}$ at an $\ell$-th root of unity is a power of $\ell$. Donkin [4] showed that this Cartan matrix has the same determinant (and the same invariant factors) as the Cartan matrix $C_{n}$ described above. Mathas' conjecture was verified for $\ell$-blocks of the Iwahori-Hecke algebras in [3]. As we shall see this implies that $\operatorname{det}(C(B))$ is a specific power of $\ell$ (depending on the weight $w(B)$ ) for each combinatorial $\ell$-block $B$ of $S_{n}$. This power may be described in two ways, see Propositions 6.10 and 6.9. We first show that the determinant $\operatorname{det}(C(B))$ calculated in [3] is the same as the one conjectured in [1]. (A proof of this is indicated at the end of [3].) Therefore the conjecture of [1] for $\operatorname{det}\left(C_{n}\right)$ also holds for arbitrary $\ell$ and based on this we may prove that the $r_{\ell}(\lambda)$ 's give the right determinant.

Let us write a partition $\lambda$ exponentially as $\lambda=\left(1^{a_{1}(\lambda)}, 2^{a_{2}(\lambda)}, \cdots\right)$. The length $l(\lambda)$ is then $\sum_{i>1} a_{i}(\lambda)$, ie. the number of parts of $\lambda$. When $\lambda$ and $\mu$ are partitions, let $\lambda+\mu=\left(i^{a_{i}(\lambda)+a_{i}(\mu)}\right)$.

Let us define the total length function $l$ by $l(n)=\sum_{\lambda \vdash n} l(\lambda)$. We refer to Section 3 for the definition of the set of $e$-quotients of $w, \mathcal{K}(e, w)$ and its cardinality $k(e, w)$.

Lemma 6.8. Let $e \in \mathbb{N}, w \geq 0$. Then

$$
\sum_{\lambda \vdash w} l(\lambda) \prod_{i \geq 1}\left(\begin{array}{c}
a_{i}(\lambda)+e \\
a_{i}(\lambda)
\end{array}\right)=(e+1) \sum_{j=0}^{w} l(j) k(e, w-j) .
$$

Proof: If $\underline{\mu}=\left(\mu_{1}, \cdots \mu_{e+1}\right) \in \mathcal{K}(e+1, w)$ and $1 \leq i \leq e+1$, we define $l_{i}(\underline{\mu})=l\left(\mu_{i}\right)$ and $l(\underline{\mu})=\sum_{i} l\left(\mu_{i}\right)$. If we divide the quotients $\underline{\mu}$ into classes according to the 
cardinality $j$ of $\mu_{i}$, we get

$$
\sum_{\underline{\mu} \in \mathcal{K}(e+1, w)} l_{i}(\underline{\mu})=\sum_{j=0}^{w} l(j) k(e, w-j),
$$

where the right hand side is independent of $i$. Adding the equations for all $i$ we get

$$
\sum_{\underline{\mu} \in \mathcal{K}(e+1, w)} l(\underline{\mu})=(e+1) \sum_{j=0}^{w} l(j) k(e, w-j) .
$$

We need the trivial combinatorial fact that the number of $(e+1)$-tuples of nonnegative integers $\left(t_{1}, \cdots, t_{e+1}\right)$ satisfying $t_{1}+\cdots+t_{e+1}=t$ is the binomial coefficient $\left(\begin{array}{c}t+e \\ t\end{array}\right)$. ¿From this we get that if $\lambda \vdash w$ then

$$
k(e+1, \lambda):=\prod_{i \geq 1}\left(\begin{array}{c}
a_{i}(\lambda)+e \\
a_{i}(\lambda)
\end{array}\right)
$$

is also the number of $(e+1)$-quotients $\underline{\mu}=\left(\mu_{1}, \cdots, \mu_{e+1}\right)$ with $\sum_{i} \mu_{i}=\lambda$. Clearly, if $\sum_{i} \mu_{i}=\lambda$, then $l(\underline{\mu})=l(\lambda)$. If we divide the summands of $(1)$ into classes according to $\lambda=\sum_{i} \mu_{i}$ we get then

$$
\sum_{\underline{\mu} \in \mathcal{K}(e+1, w)} l(\underline{\mu})=\sum_{\lambda \vdash w} l(\lambda) k(e+1, \lambda),
$$

which in view of (2) proves the lemma.

If $w \geq 0$ we define $d_{\ell}(w)=\frac{1}{\ell-1} \sum_{\lambda \vdash w} l(\lambda) \prod_{i \geq 1}\left(\begin{array}{c}a_{i}(\lambda)+\ell-2 \\ a_{i}(\lambda)\end{array}\right)$ and $c_{\ell}(w)=\sum_{j=0}^{w} l(j) k(\ell-2, w-j)$. ¿From Lemma 6.8 with $e=\ell-2$ we get:

Proposition 6.9. For all $w \geq 0$ we have $c_{\ell}(w)=d_{\ell}(w)$.

We now prove

Proposition 6.10. Let $B$ be an $\ell$-block of $S_{n}$ of weight $w$. Then $\operatorname{det}(C(B))=$ $\ell^{d_{\ell}(w)}$.

Proof: By [3], Corollary 1, the determinant of the Cartan matrix of a block of weight $w$ of the Iwahori-Hecke algebra $H_{n}$ of $S_{n}$ at an $\ell$-th root of unity is $\ell^{d_{\ell}(w)}$. By [9], Corollary 5.38, and our definition of combinatorial $\ell$-blocks of $S_{n}$ we see that $H_{n}$ and $S_{n}$ have the same number of $(\ell-)$ blocks of any given weight $w \geq 0$. By Theorem 5.10 above, the Cartan matrices of any two (combinatorial) $\ell$-blocks of symmetric groups of the same weight also have the same determinant. From [4] it follows that the $(\ell-)$ Cartan matrices of $H_{n}$ and $S_{n}$ have the same determinant. From this the proposition follows easily, by induction on $w$.

In [1] it was conjectured that if $w(B)=w$ then $\operatorname{det}(C(B))=\ell^{c_{\ell}(w)}$. Indeed the formula for $c_{\ell}(w)$ given by Theorem 3.4 in [1] is equivalent to the above in view of Proposition 2.1 of [1]. Thus Propositions 6.9 and 6.10 prove the conjecture of [1] for arbitrary $\ell$. Since $\ell$-blocks of a fixed weight have the same Cartan determinant we see that the conjecture for $\operatorname{det}\left(C_{n}\right)$ of [1] is also true. Define

$$
c_{\ell}^{*}(n)=\sum_{j \geq 1} t(j) p^{*}(n-j \ell),
$$


where $t(j)$ is the number of positive integer divisors in $j$ and as usual $p^{*}(n)$ is the number of $\ell$-(class)regular partitions of $n$. In view of the above and Theorem 3.3 of [1] we then have

Proposition 6.11. $\operatorname{det}\left(C_{n}\right)=\ell^{c_{\ell}^{*}(n)}$.

In support the above conjecture the following can be shown:

Proposition 6.12.

$$
r_{\ell}(n):=\prod_{\lambda \vdash_{\ell} n} r_{\ell}(\lambda)=\ell^{c_{\ell}^{*}(n)} .
$$

Corollary 6.13. We have $\operatorname{det}\left(C_{n}\right)=\prod_{\lambda \vdash_{\ell} n} r_{\ell}(\lambda)$

The proof of proposition 6.12 uses generating functions for convenience. We let

$$
\begin{aligned}
& P(q)=\sum_{n \geq 0} p(n) q^{n}, P^{*}(q)=\sum_{n \geq 0} p^{*}(n) q^{n}, \\
& T(q)=\sum_{n \geq 1} t(n) q^{n}, C_{\ell}^{*}(q)=\sum_{n \geq 0} c_{\ell}^{*}(n) q^{n} .
\end{aligned}
$$

Then

$$
P^{*}(q)=P(q) / P\left(q^{\ell}\right)
$$

and

$$
C_{\ell}^{*}(q)=T\left(q^{\ell}\right) P^{*}(q)
$$

We are going to need the following trivial identities:

Lemma 6.14. Let $s$ be a positive integer.

(1) $\sum_{n \geq 1} n q^{n}=\frac{q}{(1-q)^{2}}$

(2) $\sum_{n \geq 1}\lfloor n / s\rfloor q^{n}=\frac{q^{s}}{\left(1-q^{s}\right)(1-q)}$

Let

$$
T_{\ell}(q)=\sum_{\ell \nmid m} q^{m} /\left(1-q^{m}\right)
$$

the generating function for the number of divisors of $n$, which are not divisible by $\ell$. Then $T_{\ell}\left(q^{\ell^{j}}\right)$ is the generating function for the number of divisors of $n$, which are divisible by $\ell^{j}$, but not by $\ell^{j+1}$. Thus we get the identity

$$
T(q)=\sum_{j \geq 0} T_{\ell}\left(q^{\ell^{j}}\right)
$$

see also [1].

The following numbers will be important in the proof of Proposition 6.12. Fix some integers $m, t$. We assume $\ell \nmid m$, such that by definition $\ell_{m} \neq 1$. Let $p_{\ell}(m, t, n)$ be the number of $\lambda \vdash_{\ell} n$ satisfying $\left\lfloor a_{m}(\lambda) / \ell\right\rfloor=t$, i.e. $\ell t \leq a_{m}(\lambda)<\ell(t+1)$.

Lemma 6.15. We have

$$
P_{\ell}^{m, t}(q):=\sum_{n \geq 1} p_{\ell}(m, t, n) q^{n}=P^{*}(q) q^{\ell m t}\left(1-q^{\ell m}\right) .
$$


Proof: The generating function for the number of $\lambda \vdash_{\ell} n$, with $a_{m}(\lambda)=a$ is $P^{*}(q)\left(1-q^{m}\right) q^{m a}$, as is easily seen ([1]). Thus the generating function for $p_{\ell}(m, t, n)$ is $P^{*}(q)\left(1-q^{m}\right) \sum_{j=\ell t}^{\ell t+(\ell-1)} q^{m j}=P^{*}(q)\left(1-q^{m}\right) q^{\ell m t}\left(1+q^{m}+\ldots+q^{(\ell-1) m}\right)=$ $P^{*}(q) q^{\ell m t}\left(1-q^{\ell m}\right)$, as desired.

Let us factor $r_{\ell}(n)=\prod_{m} r_{\ell}^{m}(n)$, where $r_{\ell}^{m}(n)=\prod_{\lambda \vdash_{\ell} n} r_{\ell}\left(m, a_{m}(\lambda)\right)$. We note that by definition $r_{\ell}(n)$ is only divisible by primes $p \in \pi$, which is the set of primes dividing $\ell$.

Let $p \in \pi$ and assume $p^{\alpha} \top \ell$. For $0<\beta \leq \alpha$ we define $I_{\beta}=\left\{m \mid p^{\beta} \top l_{m}\right\}$. Then whenever $m \in I_{\beta}$ we have that $p \in \pi_{m}, p^{\alpha-\beta} \top(\ell, m)$ and in fact $p^{\alpha-\beta} \top m$.

For $m \in I_{\beta}$ we calculate the power of $p$ dividing $r_{\ell}^{m}(n)$, using (1) with $t=\lfloor a / \ell\rfloor$. It is $p^{e^{m}(n)}$, where $e^{m}(n)=\beta e_{1}^{m}(n)+e_{2}^{m}(n)$ and

$$
e_{1}^{m}(n)=\sum_{t \geq 1} t p_{\ell}(m, t, n), \quad e_{2}^{m}(n)=\sum_{t \geq 1} p_{\ell}(m, t, n)\left(\sum_{j \geq 1}\left\lfloor t / p^{j}\right\rfloor\right) .
$$

We have used here that the exponent to which $p$ divides $t$ ! is $\sum_{j \geq 1}\left\lfloor t / p^{j}\right\rfloor$.

Lemma 6.16. The generating functions $E_{1}^{m}(q)$ and $E_{2}^{m}(q)$ for $e_{1}^{m}(n)$ and $e_{2}^{m}(n)$ are

$$
\begin{gathered}
E_{1}^{m}(q)=P^{*}(q) \frac{q^{\ell m}}{1-q^{\ell m}} \\
E_{2}^{m}(q)=P^{*}(q) \sum_{j \geq 1} \frac{q^{\ell m p^{j}}}{1-q^{\ell m p^{j}}}
\end{gathered}
$$

Proof: By Lemma 6.15 and Lemma 6.14(1) we have

$$
E_{1}^{m}(q)=P^{*}(q)\left(1-q^{\ell m}\right) \sum_{t \geq 1} t q^{(\ell m) t}=P^{*}(q)\left(1-q^{\ell m}\right)\left(q^{\ell m} /\left(1-q^{\ell m}\right)^{2}\right),
$$

proving the first identity. By Lemma 6.15 and Lemma 6.14(2) we have

proving the second identity.

$$
\begin{aligned}
& E_{2}^{m}(q)=P^{*}(q)\left(1-q^{\ell m}\right)\left(\sum_{t, j \geq 1} p_{\ell}(m, t, n)\left\lfloor t / p^{j}\right\rfloor q^{\ell m t}\right) \\
& =P^{*}(q)\left(1-q^{\ell m}\right)\left(\sum_{j \geq 1} q^{\ell m p^{j}} /\left(1-q^{\ell m p^{j}}\right)\left(1-q^{\ell m}\right)\right),
\end{aligned}
$$

We have shown that for $m \in I_{\beta}$ we have

$$
E^{m}(n)=P^{*}(n)\left(\beta \frac{q^{\ell m}}{1-q^{\ell m}}+\sum_{j \geq 1} \frac{q^{\ell m p^{j}}}{1-q^{\ell m p^{j}}}\right)
$$

Let us for $m \in I_{\beta}$ define

$$
T^{m}(q)=\beta \frac{q^{m}}{1-q^{m}}+\sum_{j \geq 1} \frac{q^{m p^{j}}}{1-q^{m p^{j}}} .
$$

so that $E^{m}(q)=P^{*}(q) T^{m}\left(q^{\ell}\right)$. In order to prove Proposition 6.12 we need in view of (4) just to show

$$
\sum_{\beta=1}^{\alpha} \sum_{m \in I_{\beta}} T^{m}(q)=\alpha T(q)
$$


We write each $m \in I_{\beta}$ as $p^{\alpha-\beta} m^{\prime}$, where $p \nmid m^{\prime}$, and get

$$
\sum_{m \in I_{\beta}} T^{m}(q)=\beta T_{p}\left(q^{p^{\alpha-\beta}}\right)+\sum_{j>\alpha-\beta} T_{p}\left(q^{p^{j}}\right)
$$

This shows that for all $\beta, 0<\beta \leq \alpha$, the summands on the right hand side of (7) all have the form $T_{p}\left(q^{p^{j}}\right)$ for some $j \geq 0$. It is not difficult to see that each $T_{p}\left(q^{p^{j}}\right)$ occurs exactly $\alpha$ times in the sums of (7), when $\beta$ ranges from 1 to $\alpha$. Thus, using (5) above (with $\ell=p$ ) we get

$$
\sum_{\beta=1}^{\alpha} \sum_{m \in I_{\beta}} T^{m}(q)=\alpha \sum_{j \geq 0} T_{p}\left(q^{p^{j}}\right)=\alpha T(q) .
$$

Thus (6) is proved, finishing the proof of Proposition 6.12.

Acknowledgements: B. Külshammer and G.R. Robinson gratefully acknowledge financial support from an ARC-grant of the British Council and the DAAD. (Grant numbers ARC 1166 and ARC 00/27756 respectively). J.B. Olsson was supported by the Danish Natural Science Research Council. Part of this work was done while the second author visited the Newton Institute, Cambridge and while the third author was a Visiting Fellow of All Souls College, Oxford. The authors would also like to thank John Murray for providing a collection of GAP-routines for computations in symmetric groups. Further computational assistance was given by M. Schocker and R. Zimmermann.

\section{REFERENCES}

[1] C. Bessenrodt, J.B.Olsson, A note on Cartan matrices for symmetric groups, preprint 2001. To appear in Arch. Math.

[2] M. Broué Isométries parfaites, types de blocs, catégories dérivées, Astérisque 181-182 (1990), 61-92.

[3] J. Brundan, A.Kleshchev, Cartan determinants and Shapovalov forms, preprint 2001. To appear in Math. Ann.

[4] S. Donkin, Representations of Hecke algebras and characters of symmetric groups, preprint 2001. To appear in Studies in Memory of Issai Schur, edited by A. Joseph, A. Melnikov, R. Rentschler, Progress in Mathematics.

[5] G. James, The representation theory of the symmetric groups, Lecture notes in mathematics 682, Springer-Verlag 1978.

[6] G. James, A. Kerber, The representation theory of the symmetric group, Addison-Wesley 1981.

[7] A. Kerber, F. Sänger, B. Wagner, Quotienten und Kerne von Young-Diagrammen, Brettspiele und Plethysmen gewöhnlicher irreduzibler Darstellungen symmetrischer Gruppen, Mitt. Math. Sem. Giessen 149 (1981), 131-175.

[8] I.G. Macdonald, Symmetric functions and Hall polynomials, Second edition, Oxford University Press 1995.

[9] A Mathas, Iwahori-Hecke algebras and Schur algebras of the symmetric group. University Lecture Series, 15. American Mathematical Society, 1999

[10] A.O. Morris, J.B. Olsson, On q-quotients for spin characters, J.Algebra 119(1988), 51-82.

[11] J.B. Olsson, Combinatorics and representations of finite groups, Vorlesungen aus dem FB Mathematik der Univ. Essen, Heft 20, 1993.

[12] M Osima, Some remarks on characters of the symmetric groups II, Can. J. Math. 6(1954), $511-21$.

[13] M. Osima, On the representations of the generalized symmetric group I, Math. J. Okayama Univ. 4 (1954), 39-56.

[14] G.R.Robinson, Group Algebras over semi-local rings, J. Algebra 117 (1988), 409-418. 
BURKHARD KÜLSHAMMER

Mathematisches Institut

Friedrich-Schiller-Universität Jena

D-07740 Jena

Germany

kuelshammer@uni-jena.de
JØRN B. OLSSON

Matematisk Afdeling

Universitetsparken 5

DK-2100 Copenhagen

Denmark

olsson@math.ku.dk
GEOFFREY R. ROBINSON

School of Mathematics and Statistics

University of Birmingham

Birmingham B15 2TT

England

g.r.robinson@bham.ac.uk 